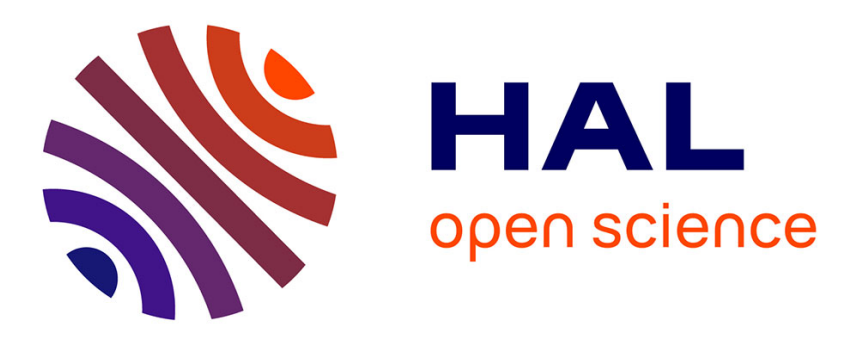

\title{
A non-local scalar conservation law describing navigation processes
}

Paulo Amorim, Florent Berthelin, Thierry Goudon

\section{To cite this version:}

Paulo Amorim, Florent Berthelin, Thierry Goudon. A non-local scalar conservation law describing navigation processes. Journal of Hyperbolic Differential Equations, 2020, 17 (4). hal-02419349

\section{HAL Id: hal-02419349 \\ https://hal.science/hal-02419349}

Submitted on 19 Dec 2019

HAL is a multi-disciplinary open access archive for the deposit and dissemination of scientific research documents, whether they are published or not. The documents may come from teaching and research institutions in France or abroad, or from public or private research centers.
L'archive ouverte pluridisciplinaire HAL, est destinée au dépôt et à la diffusion de documents scientifiques de niveau recherche, publiés ou non, émanant des établissements d'enseignement et de recherche français ou étrangers, des laboratoires publics ou privés. 


\title{
A non-local scalar conservation law describing navigation processes
}

\author{
Paulo Amorim ${ }^{1}$, Florent Berthelin ${ }^{2}$, Thierry Goudon ${ }^{2}$
}

\begin{abstract}
In this work, we consider a non-local scalar conservation law in two space dimensions which arises as the formal hydrodynamic limit of a Fokker-Planck equation. This Fokker-Planck equation is, in turn, the kinetic description of an individual-based model describing the navigation of self-propelled particles in a pheromone landscape. The pheromone may be linked to the agent distribution itself, leading to a nonlinear, nonlocal scalar conservation law where the effective velocity vector depends on the pheromone field in a small region around each point, and thus, on the solution itself. After presenting and motivating the problem, we present some numerical simulations of a closely related problem, and then prove a well-posedness and stability result for the conservation law.
\end{abstract}

\section{Introduction}

This work is mainly concerned with the scalar conservation law

$$
\partial_{t} \rho+\operatorname{div}_{x}(\rho \mathcal{U}[\rho])=0
$$

where the "velocity" $\mathcal{U}$ is defined as a non-local functional of the density $\rho$. To be more specific, the problem we are interested in naturally arises in the twodimensional framework. The model depends on two key parameters: a length $\ell>0$ and an angle $\beta \in(0, \pi / 2)$. Let us denote

$$
\Omega: \theta \in[0,2 \pi) \longmapsto \Omega(\theta)=\left(\begin{array}{c}
\cos \theta \\
\sin \theta
\end{array}\right) .
$$

We set

$$
P(t, x)=\iint_{\mathbb{R}^{2}} E(x-y) \rho(t, y) \mathrm{d} y,
$$

\footnotetext{
${ }^{1}$ Instituto de Matemática, Universidade Federal do Rio de Janeiro, Av. Athos da Silveira Ramos 149, Centro de Tecnologia - Bloco C, Cidade Universitária - Ilha do Fundão, Caixa Postal 68530, 21941-909 Rio de Janeiro, RJ - Brasil Email: paulo@im.ufrj.br. Web page: http://www.im.ufrj.br/paulo/

${ }^{2}$ Université Côte d'Azur, Inria, CNRS, LJAD, Parc Valrose, 06108 Nice, France Emails: florent.berthelin@univ-cotedazur.fr, thierry.goudon@inria.fr
} 
where $E$ is a certain convolution kernel (with respect to the space variable), and

$$
\begin{gathered}
\Psi[\rho](t, x, \theta)=\int_{0}^{\theta} \int_{0}^{\ell} \int_{\xi-\beta}^{\xi+\beta} \sin (\xi-\alpha) P(t, x+r \Omega(\alpha)) \mathrm{d} \alpha r \mathrm{~d} r \mathrm{~d} \xi, \\
\mu[\rho](t, x)=\left(\int_{0}^{2 \pi} e^{-\Psi[\rho](t, x, \theta) / D} \mathrm{~d} \theta\right)^{-1} .
\end{gathered}
$$

Finally, we have

$$
\mathcal{U}[\rho](t, x)=\mu[\rho](t, x) \int_{0}^{2 \pi} \Omega(\theta) e^{-\Psi[\rho](t, x, \theta) / D} \mathrm{~d} \theta .
$$

In the sections below we will provide appropriate motivation for these expressions.

The theory of scalar conservation laws, as far as the flux function depends on the local value of the density (namely the flux at time $t$ and position $x$ depends only on the density $\rho(t, x)$ ), is well established since the pioneering works of Kruzkov and Oleinik $[18,22]$ who, in particular, introduced relevant uniqueness criteria. The connection between these criteria is further investigated in [21], for quite general fluxes and possible source terms, still local with respect to $\rho$, but which can additionally depend on the time and space variables $t$ and $x$. The time continuity of the weak solutions is a delicate issue, for which we refer the reader to $[5,27]$. Refined estimates on the total variation of the solutions are discussed in $[7,19]$. New difficulties for the analysis arise when the flux function depends on a non local way of the unknown. Such non local fluxes have been introduced recently to model pedestrian or vehicular traffic. These difficulties are specifically addressed in $[6,7,19]$.

The model (1.1)-(1.6) is motivated by applications in life sciences: it can be seen as a hydrodynamic approximation for the modeling of self-propelled agents, the motion of which is directed by a signal they emit themselves. Here, the capability of the agents to detect the signals is embodied into the parameters $\beta$ and $\ell$. It is quite common to observe the emergence of remarkable patterns driving the motion of living organisms (like fish, birds, insects, sheep, bacteria...). Such typical organizations can occur despite the very limited communication abilities of the individuals. Many efforts have been produced to set up mathematical models that can describe the emergence of such structures $[1,13,25,8,9,14,15,23,24,26,28,29]$. Experiments can also be performed with robots [20]. Most descriptions are individual-based, and are expressed as ODEs systems. By using an approach inspired from gas dynamics, hierarchies of equations can be derived ranging from $N$-particles systems to hydrodynamic models, through kinetic equations see $[4,11,10,12,16,17]$. These macroscopic models are more amenable to numerical simulations than the individual-based coupled ODEs when the number of individuals becomes large; they are also well-adapted to bring out the collective behavior of the population. Here, we investigate these issues, considering the case where the individuals can feel the 
signal in a very limited region of space, namely, a circular sector facing the direction of their motion, delimited by the parameters $\ell, \beta$, see Fig. 1 below. Such a situation is discussed in [3], and, having in mind the specific application to the description of ants foraging, in [2], where $\beta=\pi / 2$ is shown to be critical in terms of stability of the trajectory that follows a given signal.

The paper is organized as follows. Section 2 is devoted to the modeling issues: we start from an individual-based description; then, we describe the dynamics of many interacting agents by means of a Fokker-Planck equation, and finally, the hydrodynamic regime leads to (1.1)-(1.6). Section 3 provides illustrations of the behavior of the solutions, based on numerical experiments. In Section 4, we turn to the analysis of the non-linear system: we prove the existence-uniqueness of weak solutions with bounded variation.

\section{Modeling issues}

This work is concerned with the modeling of the motion of self-propelled agents, directed according to some signal. The individuals have very limited measurements abilities; in particular they are able to feel the amplitude of the signal pointwise in a certain region but they cannot evaluate gradients. The mechanisms thus differ from the standard chemotaxis processes. In what follows, the individuals have a restricted domain of observation characterized in terms of

- a "cognitive horizon": objects located at far distance are ignored,

- a "vision cone": individuals can feel the signal only in a cone directed by the direction of their motion.

This measurement capability is therefore embodied into two key parameters $0<\ell \ll 1$ and $\beta \in(0, \pi / 2)$, see Fig. 1 .

Agent-based model. In this model, inspired from [3], each individual moves in the plane $\mathbb{R}^{2}$ at a constant speed, and the signal produces a torque, thus inducing a turning behaviour. The position/velocity pair for each individual is governed by the ODE system

$$
\left\{\begin{array}{l}
\frac{\mathrm{d}}{\mathrm{d} t} X(t)=V(t), \\
\frac{\mathrm{d}}{\mathrm{d} t} V(t)=V(t)^{\perp}\left(V(t)^{\perp} \cdot F_{X(t), V(t)}\right)
\end{array}\right.
$$

where we use the notation $V=(a, b) \in \mathbb{R}^{2} \mapsto V^{\perp}=(-b, a)$, and $F_{X, V}$ describes the reaction to the signal, for individuals located in $X$, and moving in the direction $V$. Let $x \in \mathbb{R}^{2} \mapsto P(x) \geq 0$ be the quantity that defines the signal. (For the time being we assume that $P$ is given and does not depend on the time variable.) It induces the field

$$
F_{X, V}=\int_{\mathscr{C}(V)} \frac{h}{|h|} P(X+h) \mathrm{d} h
$$




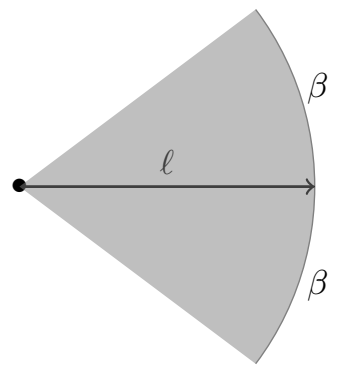

Figure 1: The domain of observation

where $\mathscr{C}(V)$ is the cone, delimited by the observation length $\ell$ and the vision angle $\beta$, as in Fig. 1:

$$
\mathscr{C}(V)=\left\{W \in \mathbb{R}^{2},|W| \leq \ell,|W \cdot V| \leq|W||V| \cos \beta\right\} .
$$

Note that the acceleration term can be equivalently rewritten as $V(t) \wedge(V(t) \wedge$ $\left.F_{X, V}\right)$. By construction the model conserves the speed $|V(t)|$, that can be fixed to 1. It is convenient to parametrize the motion through the polar angle $\theta$. Namely, we set $V(t)=\Omega(\theta(t))$ (see (1.2)). Then, we can parametrize $\mathscr{C}(V)$ by $r \in(0, \ell)$, and $\alpha \in(\theta-\beta, \theta+\beta)$, and, by using the change of variabes $h=r \Omega(\alpha)$, we rewrite

$$
F_{X, V}=\int_{0}^{\ell} \int_{\theta-\beta}^{\theta+\beta} \Omega(\alpha) P(X+r \Omega(\alpha)) r \mathrm{~d} r \mathrm{~d} \alpha .
$$

We have

$$
\frac{\mathrm{d}}{\mathrm{d} t} V(t)=V(\theta(t))^{\perp} \frac{\mathrm{d}}{\mathrm{d} t} \theta(t),
$$

and

$$
\Omega(\theta)^{\perp} \cdot \Omega(\alpha)=\sin \alpha \cos \theta-\cos \alpha \sin \theta=\sin (\alpha-\theta)
$$

so that the system (2.1) can be recast as

$$
\left\{\begin{array}{l}
\frac{\mathrm{d}}{\mathrm{d} t} X(t)=\Omega(\theta(t)) \\
\frac{\mathrm{d}}{\mathrm{d} t} \theta(t)=-\int_{0}^{\ell} \int_{\theta-\beta}^{\theta+\beta} \sin (\theta-\alpha) P(X+r \Omega(\alpha)) r \mathrm{~d} r \mathrm{~d} \alpha
\end{array}\right.
$$


It is relevant to add some "noise effect", which leads to consider instead the stochastic ODE system

$$
\left\{\begin{array}{l}
\mathrm{d} X(t)=\Omega(\theta(t)) \mathrm{d} t \\
\mathrm{~d} \theta(t)=-\left(\int_{0}^{\ell} \int_{\theta-\beta}^{\theta+\beta} \sin (\theta-\alpha) P(X+r \Omega(\alpha)) r \mathrm{~d} r \mathrm{~d} \alpha\right) \mathrm{d} t+\sqrt{2 D} \mathrm{~d} B_{t} .
\end{array}\right.
$$

We refer the reader to [3] for further comments on this model.

Kinetic model. When the number of agents is large, it becomes relevant to describe the population in terms of statistical physics, by using the particle distribution function $f(t, x, \theta): \int_{\mathcal{O}} \int_{\xi}^{\xi^{\prime}} f(t, x, \theta) \mathrm{d} \theta \mathrm{d} x$ gives the probable number of individuals which, at time $t$, have their position in the subdomain $\mathcal{O} \subset \mathbb{R}^{2}$, and are moving in a direction characterized by the angle $\theta \in\left(\xi, \xi^{\prime}\right)$. This quantity obeys the following Fokker-Planck like equation

$$
\begin{aligned}
& \partial_{t} f+\Omega(\theta) \cdot \nabla_{x} f=\frac{1}{\tau} Q(f), \\
& Q(f)=\partial_{\theta}\left(\psi(x, \theta) f+D \partial_{\theta} f\right),
\end{aligned}
$$

where the interaction force is now embodied into

$$
\psi(x, \theta)=\int_{0}^{\ell} \int_{\theta-\beta}^{\theta+\beta} \sin (\theta-\alpha) P(x+r \Omega(\alpha)) r \mathrm{~d} r \mathrm{~d} \alpha .
$$

Notice that it depends on the space variable, through the variation of the signal. The derivation of the kinetic model (2.4) from the ODEs system for $N$ individuals, as $N \rightarrow \infty$, could be justified in the mean field regime. In (2.4), up to a suitable rescaling, we have made a relaxation time $\tau>0$ appear.

In order to identify the equilibrium functions that make the interaction term $Q$ vanish, we set

$$
\Psi(x, \theta)=\int_{0}^{\theta} \psi(x, \xi) \mathrm{d} \xi
$$

and

$$
\mathscr{M}(x, \theta)=\mu(x) e^{-\Psi(x, \theta) / D}, \quad \frac{1}{\mu(x)}=\int_{0}^{2 \pi} e^{-\Psi(x, \theta) / D} \mathrm{~d} \theta .
$$

The following lemma contains some basic properties of the function $\mathscr{M}$.

Lemma 2.1. The function $\theta \mapsto \mathscr{M}(x, \theta)$ is positive, normalized (in the sense that $\left.\int_{0}^{2 \pi} \mathscr{M}(x, \theta) \mathrm{d} \theta=1\right)$, and $2 \pi$-periodic.

Proof. The first two assertions are obvious. That $\mathscr{M}(x, \theta)$ is $2 \pi$-periodic is a consequence of the fact that

$$
\int_{0}^{2 \pi} \psi(x, \theta) \mathrm{d} \theta=0
$$


This follows from an application of the Fubini theorem, see Fig. 2. Let $I$ denote the integral to be evaluated in (2.6). We have

$$
\begin{aligned}
I= & \int_{0}^{\ell} \int_{-\beta}^{2 \pi+\beta}\left(\int_{\alpha-\beta}^{\alpha+\beta} \sin (\theta-\alpha) \mathrm{d} \theta\right) P(x+r \Omega(\alpha)) r \mathrm{~d} r \mathrm{~d} \alpha \\
& -\int_{0}^{\ell} \int_{-\beta}^{\beta}\left(\int_{\alpha-\beta}^{0} \sin (\theta-\alpha) \mathrm{d} \theta\right) P(x+r \Omega(\alpha)) r \mathrm{~d} r \mathrm{~d} \alpha \\
& -\int_{0}^{\ell} \int_{2 \pi-\beta}^{2 \pi+\beta}\left(\int_{2 \pi}^{\alpha+\beta} \sin (\theta-\alpha) \mathrm{d} \theta\right) P(x+r \Omega(\alpha)) r \mathrm{~d} r \mathrm{~d} \alpha .
\end{aligned}
$$

The first integral clearly vanishes. The last integral can be rewritten as

$$
\begin{aligned}
\int_{0}^{\ell} \int_{-\beta}^{+\beta} & \left(\int_{2 \pi}^{2 \pi+\alpha+\beta} \sin (\theta-\alpha) \mathrm{d} \theta\right) P(x+r \Omega(\alpha)) r \mathrm{~d} r \mathrm{~d} \alpha \\
& =\int_{0}^{\ell} \int_{-\beta}^{+\beta}\left(\int_{0}^{\alpha+\beta} \sin (\theta-\alpha) \mathrm{d} \theta\right) P(x+r \Omega(\alpha)) r \mathrm{~d} r \mathrm{~d} \alpha
\end{aligned}
$$

so that

$$
I=\int_{0}^{\ell} \int_{-\beta}^{+\beta}\left(\int_{\alpha-\beta}^{\alpha+\beta} \sin (\theta-\alpha) \mathrm{d} \theta\right) P(x+r \Omega(\alpha)) r \mathrm{~d} r \mathrm{~d} \alpha=0
$$

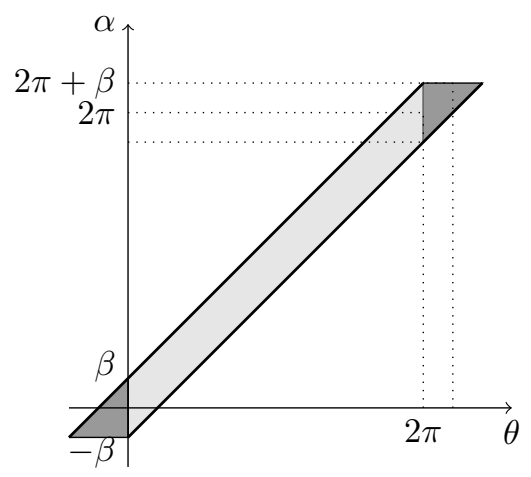

Figure 2: Integration domain

Hydrodynamic regime. The Fokker-Planck operator recasts as

$$
Q(f)=D \partial_{\theta}\left(\mathscr{M} \partial_{\theta} \frac{f}{\mathscr{M}}\right)
$$


and $Q(f)$ vanishes iff $f$ is proportional to $\mathscr{M}$. The PDE (2.4) satisfies a mass conservation property, since $\int Q(f) \mathrm{d} \theta=0$. Let

$$
\left(\begin{array}{l}
\rho \\
J
\end{array}\right)(t, x)=\int_{0}^{2 \pi}\left(\begin{array}{c}
1 \\
\Omega(\theta)
\end{array}\right) f(t, x, \theta) \mathrm{d} \theta
$$

We thus have

$$
\partial_{t} \rho+\operatorname{div}_{x} J=0 .
$$

For small relaxation times $0<\tau \ll 1$, we expect that $f(t, x, \theta) \simeq \rho(t, x) \mathscr{M}(x, \theta)$. Replacing $f$ by this ansatz in (2.7), we are led to a scalar conservation law for the density $\rho$

$$
\partial_{t} \rho+\operatorname{div}_{x}(\rho \mathcal{U})=0
$$

which involves the effective velocity

$$
\mathcal{U}(x)=\int_{0}^{2 \pi} \Omega(\theta) \mathscr{M}(x, \theta) \mathrm{d} \theta .
$$

Definition of the signal. If the signal $P$ is given, the model is linear. However, for many applications, the signal is emitted by the individuals themselves. Therefore, $P$ depends on the density of individuals. We can assume it is defined self-consistently by the diffusion equation

$$
\partial_{t} P-\Delta_{x} P=\rho,
$$

endowed with some initial condition $P(0, \cdot)$. When dealing with a finite number $N \in \mathbb{N}$ of individuals, the right hand side is nothing but $\sum_{j=1}^{N} \delta\left(x=X_{j}(t)\right)$, the $X_{j}$ 's being defined by the ODEs (2.1), or the stochastic version (2.3).

Strictly speaking, the solution of (2.8) is not of the form (1.3), since it is represented by a convolution with a kernel in time as well as in space. In the numerical experiments that follow, we still use the physically relevant form (2.8), even though we restrict the analysis in Section 4 to the case where the signal $P$ has the form (1.3).

A simplified asymptotic model. In order to simplify the computation, it is tempting to use the truncated expansion

$$
P(x+r \Omega(\alpha)) \simeq P(x)+r \nabla_{x} P(x) \cdot \Omega(\alpha)+\frac{r^{2}}{2} D_{x}^{2} P(x): \Omega(\alpha) \otimes \Omega(\alpha) .
$$

It leads to replace $\psi(x, \theta)$ in (2.5) by the following expansion

$$
\psi_{0}(x, \theta)+\psi_{1}(x, \theta)+\psi_{2}(x, \theta)
$$

based on the zeroth, first, and second order terms with respect to $P$. We shall similarly express the approached equilibrium function in (1.4) under the form

$$
\Psi_{0}(x, \theta)+\Psi_{1}(x, \theta)+\Psi_{2}(x, \theta) .
$$


We start by observing that

$$
\psi_{0}(x, \theta)=P(x) \int_{0}^{\ell} \int_{\theta-\beta}^{\theta+\beta} \sin (\theta-\alpha) r \mathrm{~d} r \mathrm{~d} \alpha=0,
$$

and we set $\Psi_{0}(x, \theta)=0$ as well. Next, we shall use the basic identity

$$
\begin{aligned}
\Omega(\alpha) \sin (\theta-\alpha) & =-\Omega(\alpha) \Omega(\alpha) \cdot \Omega(\theta)^{\perp}=-\Omega(\alpha) \otimes \Omega(\alpha) \Omega(\theta)^{\perp} \\
& =-\frac{1}{2}\left(\mathbb{I}+\left(\begin{array}{cc}
\cos (2 \alpha) & \sin (2 \alpha) \\
\sin (2 \alpha) & -\cos (2 \alpha)
\end{array}\right)\right) \Omega(\theta)^{\perp} .
\end{aligned}
$$

It leads to

$$
\begin{aligned}
& \psi_{1}(x, \theta) \\
& \qquad=-\nabla_{x} P(x) \cdot \int_{0}^{\ell} \int_{\theta-\beta}^{\theta+\beta} \frac{1}{2}\left(\mathbb{I}+\left(\begin{array}{cc}
\cos (2 \alpha) & \sin (2 \alpha) \\
\sin (2 \alpha) & -\cos (2 \alpha)
\end{array}\right)\right) \Omega(\theta)^{\perp} r^{2} \mathrm{~d} r \mathrm{~d} \alpha \\
& \quad=-\frac{\ell^{3}}{12} \nabla_{x} P(x) \cdot\left[4 \beta \mathbb{I}+\left(\begin{array}{cc}
C & S \\
S & -C
\end{array}\right)\right] \Omega(\theta)^{\perp}
\end{aligned}
$$

with

$$
C=\sin (2(\theta+\beta))-\sin (2(\theta-\beta)), \quad S=\cos (2(\theta-\beta))-\cos (2(\theta+\beta)) .
$$

We thus get

$$
\begin{aligned}
\psi_{1}(x, \theta) & =\frac{\ell^{3}}{12}\left[-4 \beta \Omega(\theta)^{\perp}+\left(\begin{array}{c}
\cos (\theta+2 \beta)-\cos (\theta-2 \beta) \\
\sin (\theta+2 \beta)-\sin (\theta-2 \beta)
\end{array}\right)\right] \cdot \nabla_{x} P(x) \\
& =\frac{\ell^{3}}{6}(\sin (2 \beta)-2 \beta) \Omega(\theta)^{\perp} \cdot \nabla_{x} P(x)
\end{aligned}
$$

and from $\Psi_{1}(x, \theta)=\int_{0}^{\theta} \psi_{1}(x, \xi) d \xi$,

$$
\Psi_{1}(x, \theta)=\frac{\ell^{3}}{6}(\sin (2 \beta)-2 \beta)\left(\begin{array}{c}
\cos \theta-1 \\
\sin \theta
\end{array}\right) \cdot \nabla_{x} P(x) .
$$

Finally, we compute

$$
\begin{aligned}
\psi_{2}(x, \theta)= & \frac{1}{2} \int_{0}^{\ell} \int_{\theta-\beta}^{\theta+\beta}(\sin \theta \cos \alpha-\cos \theta \sin \alpha) \\
& \times\left(\begin{array}{cc}
\cos ^{2} \alpha & \sin \alpha \cos \alpha \\
\sin \alpha \cos \alpha & \sin ^{2} \alpha
\end{array}\right) r^{3} \mathrm{~d} r \mathrm{~d} \alpha: D_{x}^{2} P(x) \\
= & \frac{\ell^{4}}{8} \sin \theta \int_{\theta-\beta}^{\theta+\beta}\left(\begin{array}{cc}
\cos ^{3} \alpha & -\frac{1}{3} \frac{\mathrm{d}}{\mathrm{d} \alpha}\left[\cos ^{3} \alpha\right] \\
-\frac{1}{3} \frac{\mathrm{d}}{\mathrm{d} \alpha}\left[\cos ^{3} \alpha\right] & \frac{1}{3} \frac{\mathrm{d}}{\mathrm{d} \alpha}\left[\sin ^{3} \alpha\right]
\end{array}\right) \mathrm{d} \alpha: D_{x}^{2} P(x) \\
& -\frac{\ell^{4}}{8} \cos \theta \int_{\theta-\beta}^{\theta+\beta}\left(\begin{array}{cc}
-\frac{1}{3} \frac{\mathrm{d}}{\mathrm{d} \alpha}\left[\cos ^{3} \alpha\right] & \frac{1}{3} \frac{\mathrm{d}}{\mathrm{d} \alpha}\left[\sin ^{3} \alpha\right] \\
\frac{1}{3} \frac{\mathrm{d}}{\mathrm{d} \alpha}\left[\sin ^{3} \alpha\right] & \sin ^{3} \alpha
\end{array}\right) \mathrm{d} \alpha: D_{x}^{2} P(x)
\end{aligned}
$$


and obtain after a lengthy but standard computation,

$$
\psi_{2}(x, \theta)=\frac{\ell^{4}}{8}\left(a(\theta) \partial_{11}^{2} P(x)+b(\theta) \partial_{12}^{2} P(x)+c(\theta) \partial_{22}^{2} P(x)\right)
$$

with

$$
\begin{aligned}
& a(\theta)=\frac{2}{3} \sin ^{3} \beta \sin 2 \theta, \\
& b(\theta)=-\frac{4}{3} \sin ^{3} \beta \cos 2 \theta, \\
& c(\theta)=-\frac{2}{3} \sin ^{3} \beta \sin 2 \theta .
\end{aligned}
$$

So, from $\Psi_{2}(x, \theta)=\int_{0}^{\theta} \psi_{2}(x, \xi) d \xi$ we get

$$
\begin{aligned}
\Psi_{2}(x, \theta)= & \frac{\ell^{4}}{24} \sin ^{3} \beta\left[(1-\cos (2 \theta)) \partial_{11}^{2} P(x)\right. \\
& \left.\quad-2 \sin (2 \theta) \partial_{12}^{2} P(x)+(\cos (2 \theta)-1) \partial_{22}^{2} P(x)\right] .
\end{aligned}
$$

\section{$3 \quad$ Numerical simulations}

In order to guide the intuition about the model and the behavior of the solutions, we provide a few simulations. The computational domain is the square $[0,1] \times$ $[0,1]$, endowed with periodic boundary conditions. When necessary, integrals with respect to $\theta$ are evaluated either with the rectangular rule, or with the third order Simpson's rule. We work with a Cartesian grid with steps $\Delta x$ (resp. $\Delta y$ ) in the horizontal (resp. vertical) direction. The conservation law is approximated by using a standard first order scheme

$$
\rho_{i, j}^{n+1}=\rho_{i, j}^{n}-\frac{\Delta t}{\Delta x}\left(F_{i+1 / 2, j}-F_{i-1 / 2, j}\right)-\frac{\Delta t}{\Delta y}\left(G_{i, j+1 / 2}-G_{i, j-1 / 2}\right) .
$$

Denoting $U, V$ the components of the velocity field, and $s^{ \pm}=\frac{s \pm|s|}{2}$, we use the upwind numerical fluxes

$F_{i+1 / 2, j}=U_{i+1 / 2, j}^{+} \rho_{i, j}^{n}+U_{i+1 / 2, j}^{-} \rho_{i+1, j}^{n}, \quad G_{i, j+1 / 2}=V_{i, j+1 / 2}^{+} \rho_{i, j}^{n}+V_{i, j+1 / 2}^{-} \rho_{i, j+1}^{n}$.

The time step $\Delta t$ is fixed by taking into account the CFL condition

$$
\Delta t \leq \max \left(\frac{\left|U_{i+1 / 2, j}\right|}{\Delta x}, \frac{\left|V_{i, j+1 / 2}\right|}{\Delta y}\right)
$$

that preserves the positivity of the solution.

In order to reduce the computational cost, we only use the simplified model where $\Psi$ is replaced by a linear expression in $\nabla_{x} P, D_{x}^{2} P$, as explained in the previous section. We shall briefly discuss the linear case where $P$ is given, so that the expression of $\nabla_{x} P, D_{x}^{2} P$ at the discretization points can be explicitely 
computed. When $P$ is defined self-consistently by (2.8), we use the implicit scheme

$$
P_{i j}^{n+1}+\Delta t\left(\Delta^{d} P^{n+1}\right)_{i j}=P_{i j}^{n}+\Delta t \rho_{i, j}^{n}
$$

where $\Delta^{d}$ is the standard 5 points discrete Laplacian operator. Then, we obtain the approximations of the gradient and the Hessian of $P$ by using finite difference operators.

\subsection{Given signal}

We start with a simulation where the signal is given:

$$
P\left(x_{1}, x_{2}\right)=e^{-\left|x_{1}-1 / 2\right|^{2} \delta},
$$

with $\delta=1 / 500$ see Fig. 3. The vision domain is defined by $\ell=1 / 10$, and $\beta=\pi / 3$. The signal attracts the individuals towards the axis $x_{1}=1 / 2$, see the velocity field in Fig. 4-left. Close to the critical axis, the horizontal components of the effective velocity field tends to 0 . The vertical component should vanish, but numerical errors can be sensitive, that here produce a spurious vertical motion (that mainly depends on the quadrature rule used to compute the angular integrals), see Fig. 4-right. The initial data is made of two bumps centered at $x=(1 / 4,1 / 4)$ and $x=(3 / 4,3 / 4)$. Since the signal is very peaked, individuals which are too far from the center of the signal cannot feel it and do not move at all. The other individuals are attracted towards the axis $x_{1}=1 / 2$. See Fig. 5 .

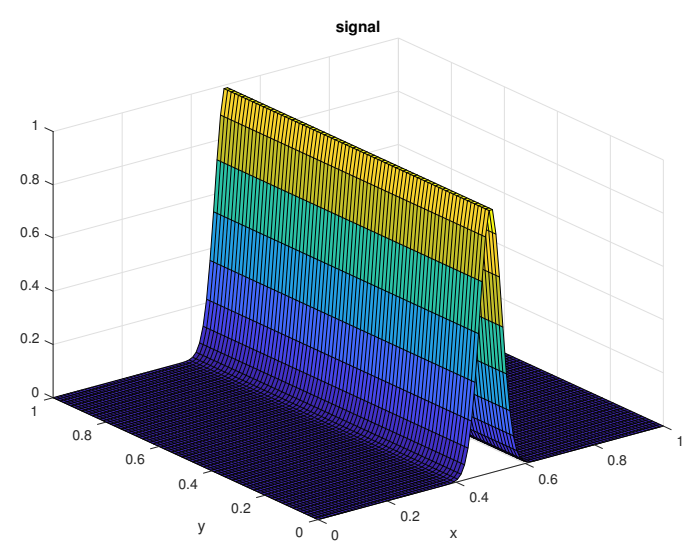

Figure 3: Given signal peaked on the axis $x=1 / 2$

The second test case with a given signal deals with

$$
P(x)=e^{-\left|r-r_{0}\right|^{2} / \delta}
$$

with $r=\sqrt{x_{1}^{2}+x_{2}^{2}}, r_{0}=.05, \delta=1 / 1000$, see Fig. 6 . Horizontal and vertical velocities are depicted in Fig. 7. We observe that the individuals are attracted 

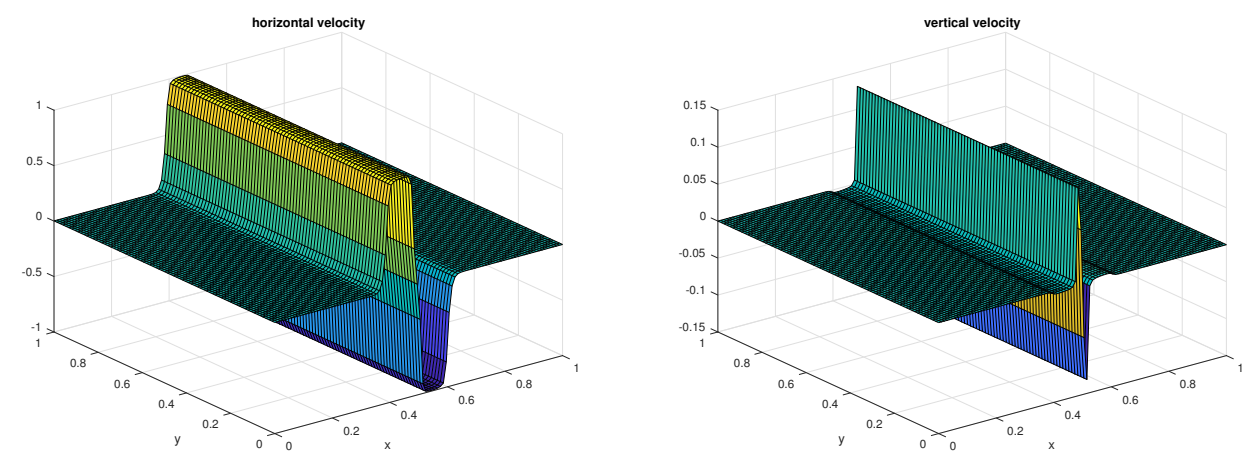

Figure 4: Given signal peaked on the axis $x=1 / 2$ : Horizontal velocity (left) and vertical velocity (right)

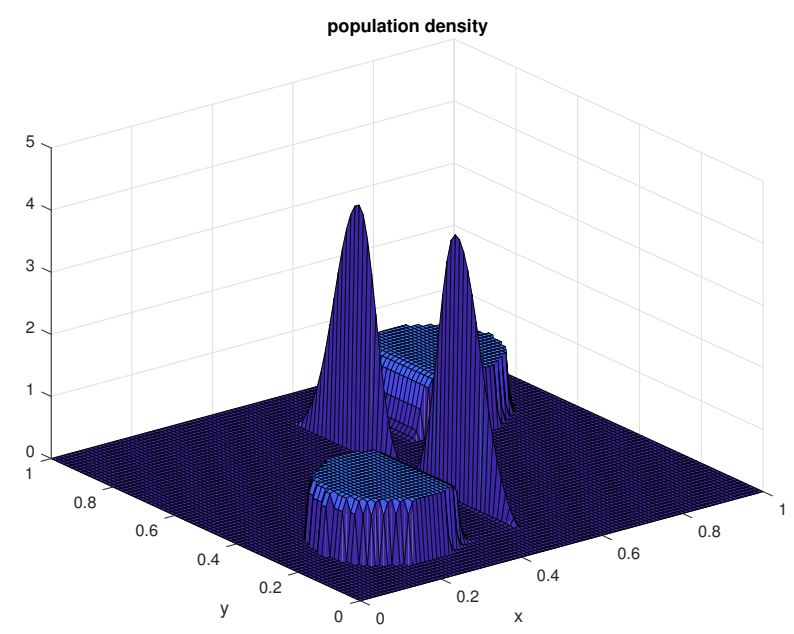

Figure 5: Given signal peaked on the axis $x=1 / 2$ : solution at $T=1.128$

towards the ring of high density, where the get stuck since there is no tangential velocity.

\subsection{Self-consistent model}

We turn to cases where the signal is produced by the individuals, according to the diffusion equation (2.8). The first case, referred to as Test 1, considers an initial data with two bumps for the individuals and a vanishing concentration of signal. We see that individuals just concentrate to the center of mass of each 


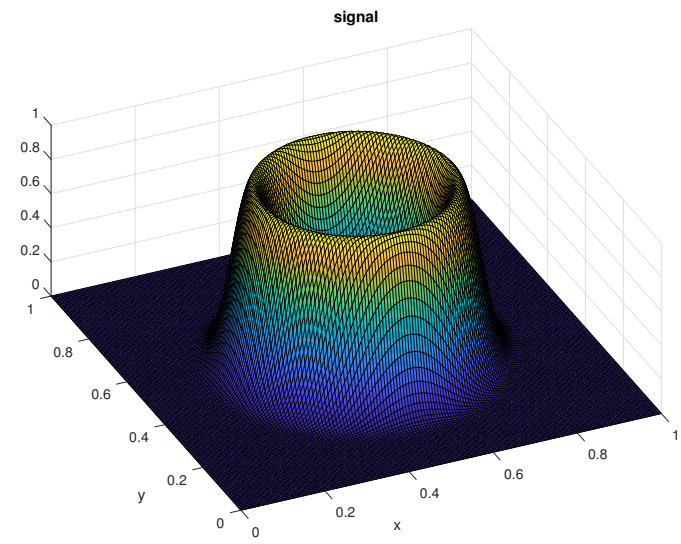

Figure 6: Given signal concentrated on a ring
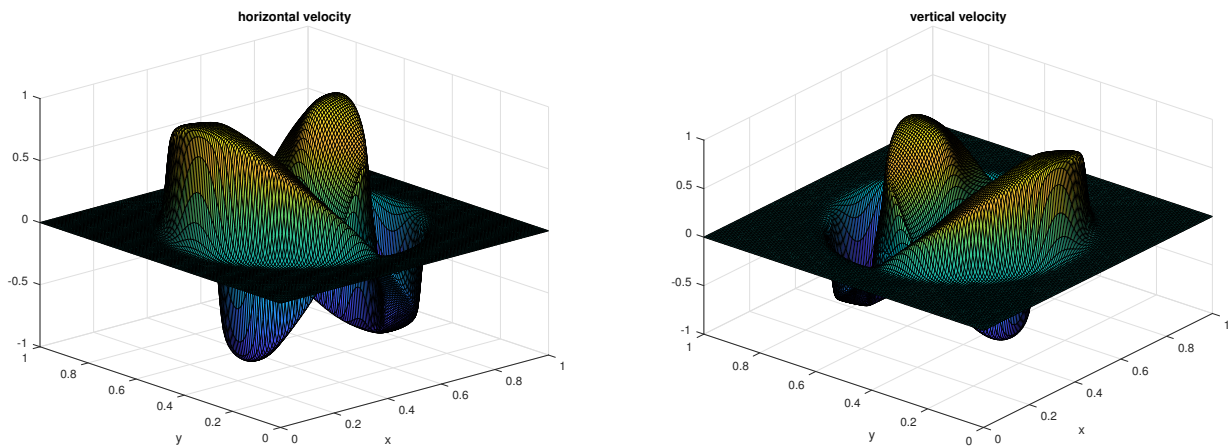

Figure 7: Given signal concentrated on a ring: Horizontal velocity (left) and vertical velocity (right)

bump, see Fig. 11-13.

The second case, Test 2, has a different flavor in order to capture a dynamics more interesting than the pure aggregation. The initial density of individuals is set uniformly to 1 , but we give an initial signal, that follows the axis $x_{1}=1 / 2$. We see in Fig. 15-16 that the individuals organize to follow the initial trail, even if the initial effect is dissipated by the diffusion equation.

We perform a similar simulation, with an initial signal making a ring. The results of this Test 3 are depicted in Fig. 17-18.

In the previous numerical tests, we can see that the main feature of the solutions of (1.1)-(1.6) (with (2.8)) is the aggregation of the population in the regions where signal density is highest. In this sense, true trail following behavior is not explicitly observed (in the sense of movement along the crest of a signal 


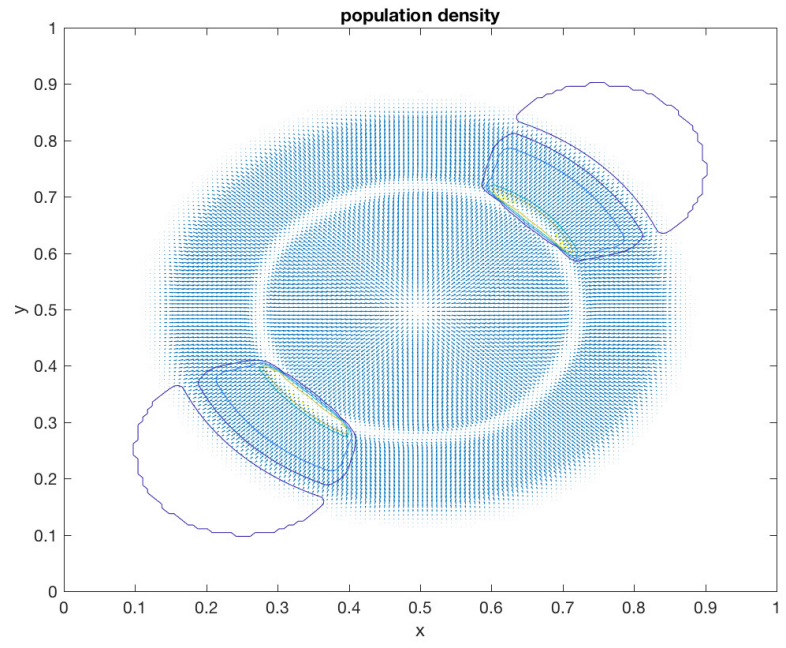

Figure 8: Given signal concentrated on a ring: solution at $T=.05$
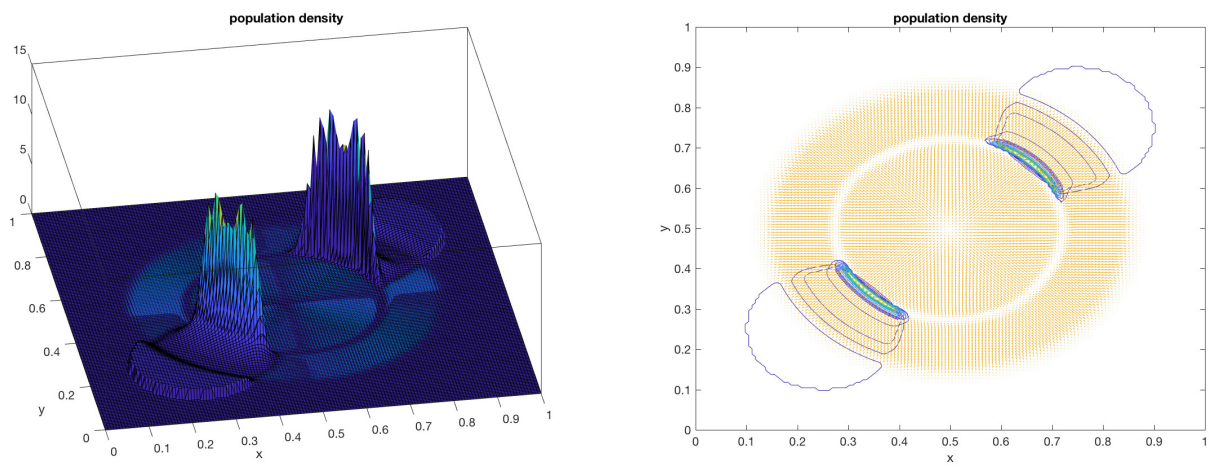

Figure 9: Given signal concentrated on a ring: solution at $T=.1$

distribution). However, this is to be expected from a first order model, since the introduction of the equilibrium distributions involve averages in the velocities; thus the description used here loses some information about microscopic velocities which might "cancel out" when passing to a macroscopic regime. This is further reinforced by the fact that the critical value $\beta=\pi / 2$ of the sensing area half-angle (above which trail following was observet to fail in [2]) does not seem to play a role in our analysis. Still, the observed behavior is not entirely reducible to an aggregation phenomena of chemotaxis type, since (as the analysis below will show), there is no finite-time blow-up phenomena, in contrast to many two-dimensional models of chemotaxis. 

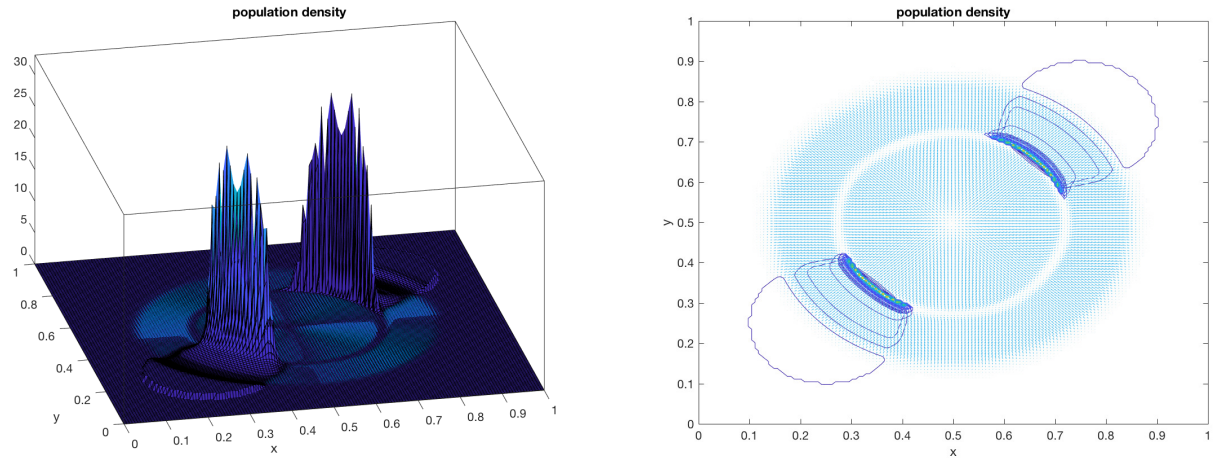

Figure 10: Given signal concentrated on a ring: solution at $T=.19$
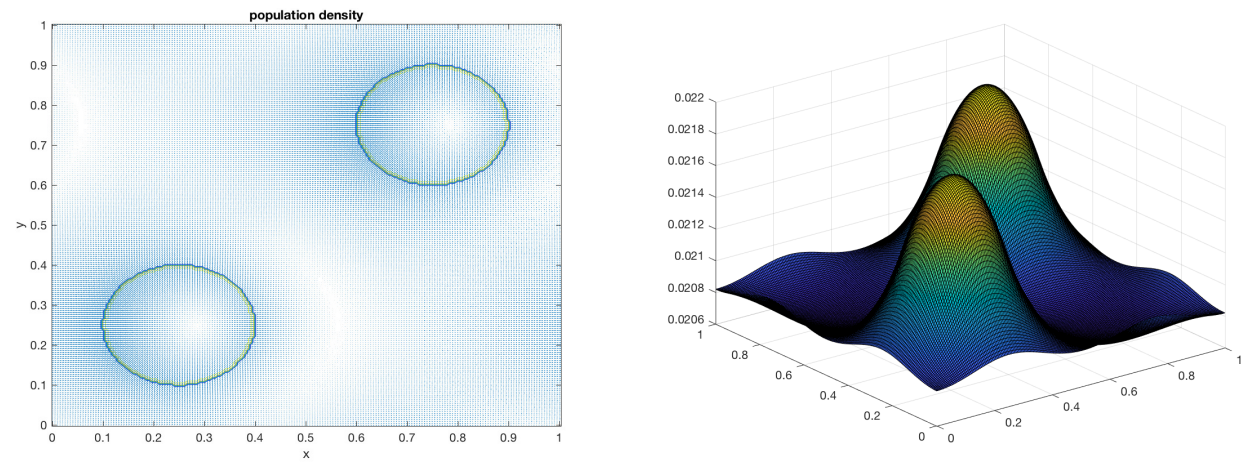

Figure 11: Coupled model, test 1: initial state (left) and velocity field
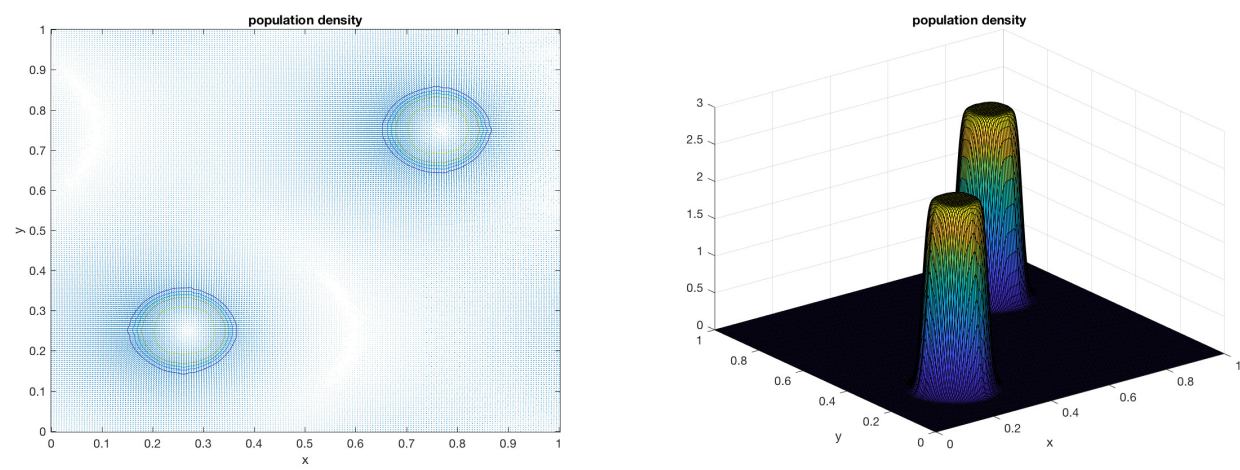

Figure 12: Coupled model, test 1: solution at time $t=13.3$ 

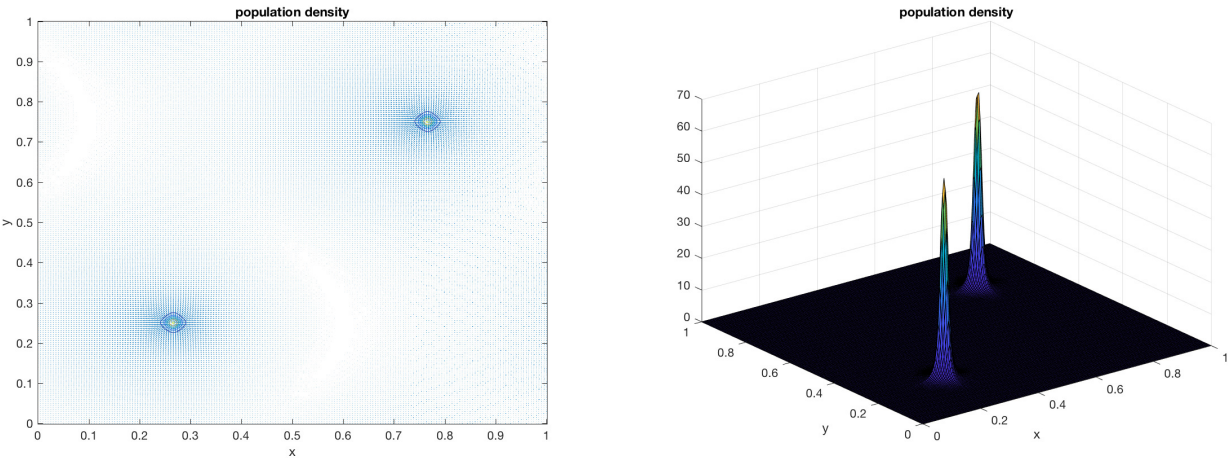

Figure 13: Coupled model, test 1: solution at time $t=20$

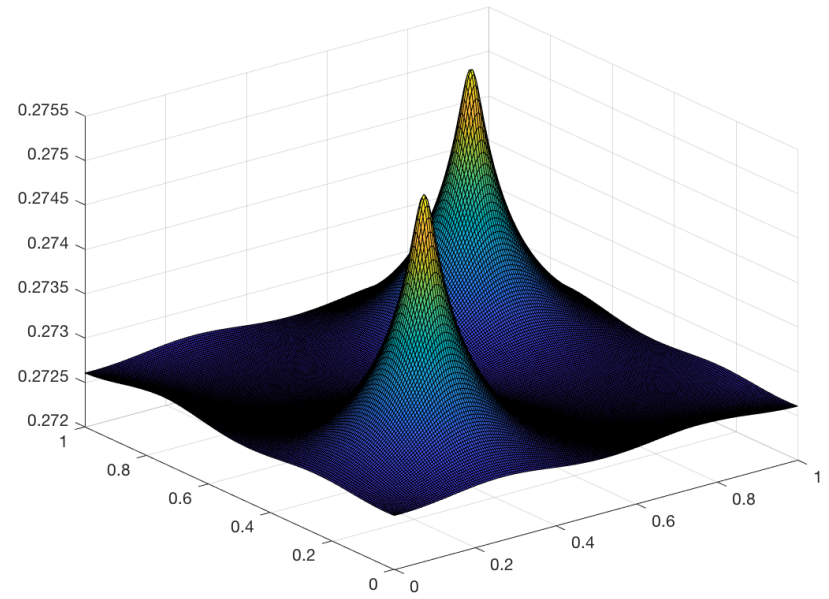

Figure 14: Coupled model, test 1: velocity field at time $t=20$

\section{Analysis of the scalar conservation law}

We turn to the analysis of the conservation law (1.1)-(1.6). We slightly simplify the coupling compared to (2.8), restricting the analysis to a mere convolution (1.3), with a smooth kernel $E$. Namely, we suppose

$$
E \in C^{2}\left(\mathbb{R}^{2}, \mathbb{R}\right) \text { with } \partial^{\alpha} E \in L^{\infty}\left(\mathbb{R}^{2}\right) \text { for any } \alpha \in \mathbb{N}^{2},|\alpha| \leq 2 \text {. }
$$

For further purposes, we denote

$$
\begin{array}{lll}
\mathcal{E}=\|E\|_{L^{\infty}\left(\mathbb{R}^{2}\right)}, & \mathcal{E}_{1}^{\prime}=\left\|\partial_{x_{1}} E\right\|_{L^{\infty}\left(\mathbb{R}^{2}\right)}, & \mathcal{E}_{2}^{\prime}=\left\|\partial_{x_{2}} E\right\|_{L^{\infty}\left(\mathbb{R}^{2}\right)}, \\
\mathcal{E}_{11}^{\prime \prime}=\left\|\partial_{x_{1} x_{1}}^{2} E\right\|_{L^{\infty}\left(\mathbb{R}^{2}\right)}, & \mathcal{E}_{22}^{\prime \prime}=\left\|\partial_{x_{2} x_{2}}^{2} E\right\|_{L^{\infty}\left(\mathbb{R}^{2}\right)}, & \mathcal{E}_{12}^{\prime \prime}=\left\|\partial_{x_{1} x_{2}}^{2} E\right\|_{L^{\infty}\left(\mathbb{R}^{2}\right)},
\end{array}
$$



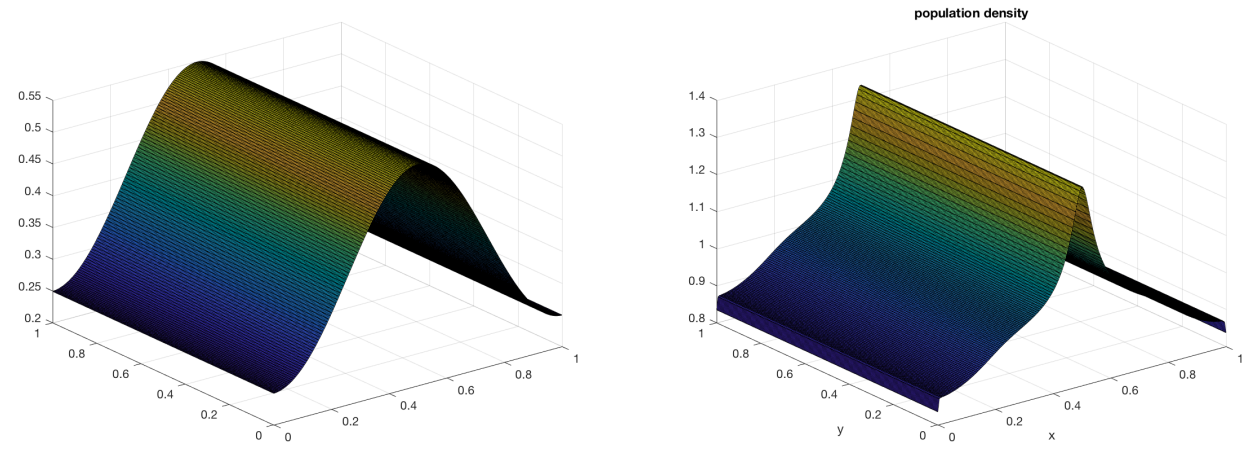

Figure 15: Coupled model, test 2: signal and solution at time $t=.03$
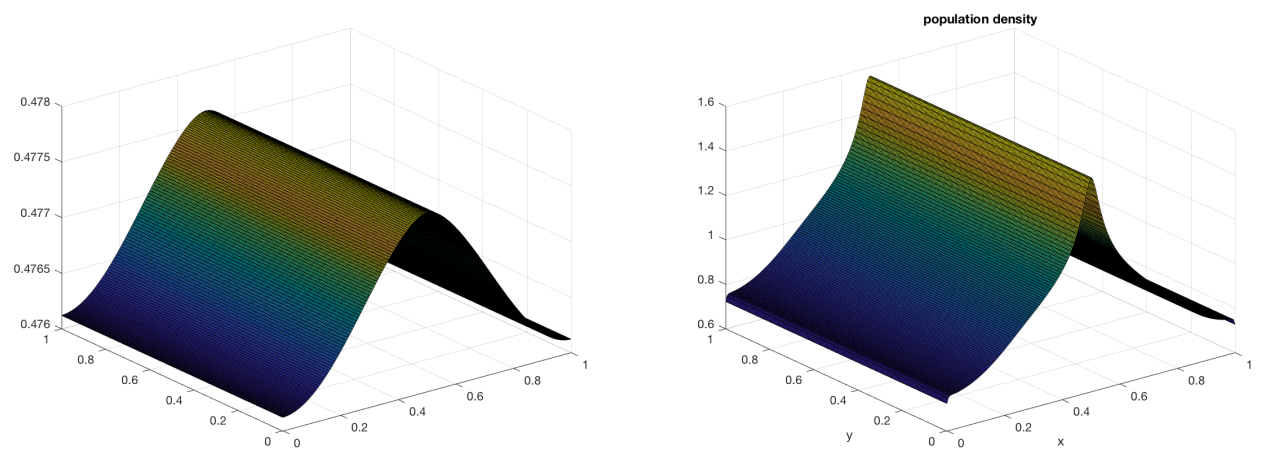

Figure 16: Coupled model, test 2: signal and solution at time $t=.3$
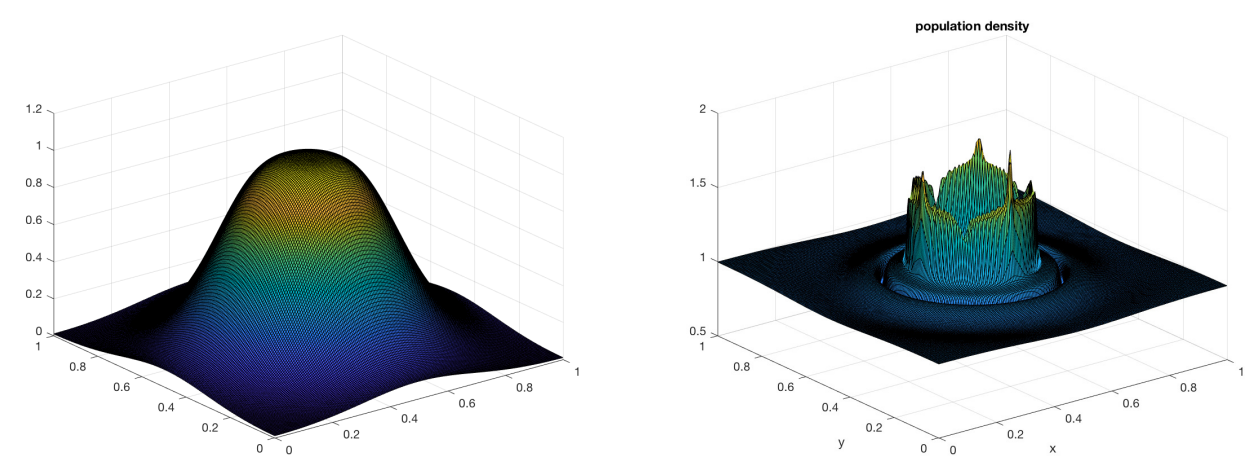

Figure 17: Coupled model, test 3: ignal and solution at time $t=.01$ 

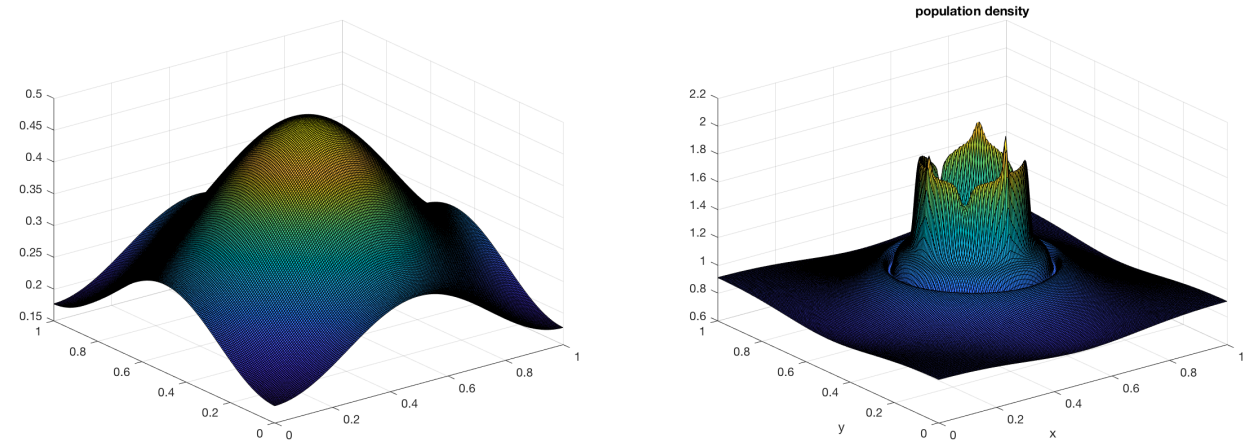

Figure 18: Coupled model, test 3: signal and solution at time $t=.04$

and

$$
c=\frac{(2 \pi \ell)^{2}}{D} .
$$

The main result states as follows.

Theorem 4.1. Assume $(\mathbf{H})$ and let $\rho_{0} \in L^{1} \cap L^{\infty} \cap B V\left(\mathbb{R}^{2}\right)$. Then, there exists a unique $\rho \in C^{0}\left([0, \infty) ; L^{1}\left(\mathbb{R}^{2}\right)\right)$, which also lies in $L^{\infty}\left(0, T ; B V\left(\mathbb{R}^{2}\right)\right) \cap$ $L^{\infty}\left((0, T) \times \mathbb{R}^{2}\right)$ for any $0<T<\infty$, weak entropy solution of $(1.1)-(1.6)$ associated to the initial data $\rho_{0}$.

Inspired from [6, 7], we proceed according to the following steps:

- for $0<T<\infty$ and a given $\sigma \in C^{0}\left([0, T] ; L^{1}\left(\mathbb{R}^{2}\right)\right)$, we consider the weak solution of the linear problem

$$
\begin{aligned}
& \partial_{t} \rho+\operatorname{div}_{x}(\rho \mathcal{U}[\sigma])=0 \quad \text { on }(0, T) \times \mathbb{R}^{2}, \\
& \left.\rho\right|_{t=0}=\rho_{0} .
\end{aligned}
$$

- this defines a mapping

$$
\Gamma: \sigma \longmapsto \rho,
$$

the functional properties of which rely on the estimates satisfied by $\sigma \mapsto$ $\mathcal{U}[\sigma]$.

- provided $T$ is small enough, we justify that $\Gamma$ admits a unique fixed point in a suitable Banach space $X_{T}$.

- it remains to justify that the solution has an infinite lifespan, and that it satisfies the entropy criterion. This relies on further estimates of the total variation of the solutions. 


\subsection{Preliminary estimates}

We shall need several estimates, in $L^{\infty}$ and $L^{1}$ norms on the non local coefficients and their space derivatives. The proofs are postponed to Appendix A.

Lemma 4.2 ( $L^{\infty}$ estimate). Let $(\mathbf{H})$ be fulfiled. Let $\sigma \in L^{1}\left(\mathbb{R}^{2}\right)$. Then, the following estimates hold:

$$
\begin{aligned}
& |\Psi[\sigma](x, \theta)| \leq c D \mathcal{E}\|\sigma\|_{L^{1}}, \\
& e^{-c \mathcal{E}\|\sigma\|_{L^{1}}} \mid \leq e^{-\Psi[\sigma](x, \theta) / D} \leq e^{c \mathcal{E}\|\sigma\|_{L^{1}}}, \\
& |\mu[\sigma](x)| \leq \frac{e^{c \mathcal{E}\|\sigma\|_{L^{1}}}}{2 \pi} ; \\
& \left|\partial_{x_{k}} \Psi[\sigma](x, \theta)\right| \leq c D \mathcal{E}_{k}^{\prime}\|\sigma\|_{L^{1}}, \\
& \left|\partial_{x_{k}} \mu[\sigma](x)\right| \leq \frac{c \mathcal{E}_{k}^{\prime}\|\sigma\|_{L^{1}}}{2 \pi} e^{3 c \mathcal{E}\|\sigma\|_{L^{1}}} \\
& \left|\partial_{x_{k} x_{m}}^{2} \Psi[\sigma](x, \theta)\right| \leq c^{2} D^{2} \mathcal{E}_{k m}^{\prime \prime}\|\sigma\|_{L^{1}}, \\
& \left|\partial_{x_{k} x_{m}}^{2} \mu[\sigma](x)\right| \leq \frac{1}{2 \pi}\left(c \mathcal{E}_{k m}^{\prime \prime}\|\sigma\|_{L^{1}}+3 c^{2} \mathcal{E}_{k}^{\prime} \mathcal{E}_{m}^{\prime}\|\sigma\|_{L^{1}}^{2}\right) e^{5 c \mathcal{E}\|\sigma\|_{L^{1}}} .
\end{aligned}
$$

Lemma 4.3 ( $L^{1}$ estimate). Let $(\mathbf{H})$ be fulfiled. Let $\sigma \in L^{1}\left(\mathbb{R}^{2}\right)$. Then, the following estimates hold:

$$
\begin{aligned}
& \iint_{\mathbb{R}^{2}}|\Psi[\sigma](x, \theta)| \mathrm{d} x \leq c D\|E\|_{L^{1}\left(\mathbb{R}^{2}\right)}\|\sigma\|_{L^{1}\left(\mathbb{R}^{2}\right)}, \\
& \iint_{\mathbb{R}^{2}}\left|\partial_{x_{k}} \Psi[\sigma](x, \theta)\right| \mathrm{d} x \leq c D\left\|\partial_{x_{k}} E\right\|_{L^{1}\left(\mathbb{R}^{2}\right)}\|\sigma\|_{L^{1}\left(\mathbb{R}^{2}\right)} \\
& \iint_{\mathbb{R}^{2}}\left|\partial_{x_{k}} \mu[\sigma](x)\right| \mathrm{d} x \leq \frac{c}{2 \pi} e^{3 c \mathcal{E}\|\sigma\|_{L^{1}\left(\mathbb{R}^{2}\right)}\left\|\partial_{x_{k}} E\right\|_{L^{1}\left(\mathbb{R}^{2}\right)}\|\sigma\|_{L^{1}\left(\mathbb{R}^{2}\right)}} \\
& \iint_{\mathbb{R}^{2}}\left|\partial_{x_{k} x_{m}}^{2} \Psi[\sigma](x, \theta)\right| \mathrm{d} x \leq c^{2} D^{2}\left\|\partial_{x_{k} x_{m}}^{2} E\right\|_{L^{1}\left(\mathbb{R}^{2}\right)}\|\sigma\|_{L^{1}\left(\mathbb{R}^{2}\right)} \\
& \iint_{\mathbb{R}^{2}}\left|\partial_{x_{k} x_{m}}^{2} \mu[\sigma](x)\right| \mathrm{d} x \leq c^{2} C_{k m} e^{5 c \mathcal{E}\|\sigma\|_{L^{1}\left(\mathbb{R}^{2}\right)}\|\sigma\|_{L^{1}\left(\mathbb{R}^{2}\right)},}
\end{aligned}
$$

where

$C_{k m}=\frac{D}{2 \pi}\left\|\partial_{x_{k} x_{m}}^{2} E\right\|_{L^{1}\left(\mathbb{R}^{2}\right)}+\left(D\left\|\partial_{x_{k}} E\right\|_{L^{1}\left(\mathbb{R}^{2}\right)} \varepsilon_{m}^{\prime}+\frac{1}{\pi}\left\|\partial_{x_{l}} E\right\|_{L^{1}\left(\mathbb{R}^{2}\right)} \varepsilon_{k}^{\prime}\right)\|\sigma\|_{L^{1}\left(\mathbb{R}^{2}\right)}$.

\subsection{On the solutions of the linear problem (4.1)}

Next, we consider the Cauchy problem (4.1), with $\sigma:(0, \infty) \times \mathbb{R}^{2} \rightarrow \mathbb{R}$ a given function. This is nothing but a transport equation with smooth coefficients. 
Lemma 4.4. Let $\sigma \in C\left(\left[0,+\infty\left[; L^{1}\left(\mathbb{R}^{2}\right)\right) \cap L^{\infty}\left(0,+\infty ; L^{1}\left(\mathbb{R}^{2}\right)\right)\right.\right.$. Then $\mathcal{U}[\sigma]$ is a continuous function and lies in $L^{\infty}\left(0,+\infty ; W^{1, \infty}\left(\mathbb{R}^{2}\right)\right)$. We have, for any $(t, x) \in\left[0,+\infty\left[\times \mathbb{R}^{2}\right.\right.$,

$$
\|\mathfrak{U}[\sigma](t, x)\| \leq e^{2 c \mathcal{E}\|\sigma\|_{L^{\infty}\left(0, \infty ; L^{1}\left(\mathbb{R}^{2}\right)\right)}}
$$

and

$$
\left\|\partial_{x_{k}} \mathcal{U}[\sigma](t, x)\right\| \leq 2 c \mathcal{E}_{k}^{\prime}\|\sigma\|_{L^{\infty}\left(0, \infty ; L^{1}\left(\mathbb{R}^{2}\right)\right)} e^{4 c \mathcal{E}\|\sigma\|_{L^{\infty}\left(0, \infty ; L^{1}\left(\mathbb{R}^{2}\right)\right)},}
$$

for $k=1,2$.

Proof. The continuity of $\mathcal{U}[\sigma]$ is proven by direct inspection and application of the Lebesgue theorem. The first estimate follows from (4.2) in Lemma 4.2. Next, we observe that

$$
\begin{aligned}
\partial_{x_{k}} \mathcal{U}[\sigma](t, x)= & \partial_{x_{k}} \mu[\sigma](t, x) \int_{0}^{2 \pi} \cos (\theta) e^{-\Psi[\sigma](t, x, \theta) / D} \mathrm{~d} \theta \\
& -\frac{1}{D} \mu[\sigma](t, x) \int_{0}^{2 \pi} \cos (\theta) \partial_{x_{k}} \Psi[\sigma](t, x, \theta) e^{-\Psi[\sigma](t, x, \theta) / D} \mathrm{~d} \theta
\end{aligned}
$$

By using (4.3) in Lemma 4.2, we obtain

$$
\begin{aligned}
\left\|\partial_{x_{k}} \mathcal{U}[\sigma](t, x)\right\| \leq & c \mathcal{E}_{k}^{\prime}\|\sigma(t, \cdot)\|_{L^{1}\left(\mathbb{R}^{2}\right)} e^{4 c \mathcal{E}\|\sigma(t, \cdot)\|_{L^{1}\left(\mathbb{R}^{2}\right)}} \\
& +c \mathcal{E}_{k}^{\prime}\|\sigma(t, \cdot)\|_{L^{1}\left(\mathbb{R}^{2}\right)} e^{2 c \mathcal{E}\|\sigma(t, \cdot)\|_{L^{1}\left(\mathbb{R}^{2}\right)}}
\end{aligned}
$$

which proves (4.11).

Proposition 4.5. Let $\rho^{0} \in L^{1}\left(\mathbb{R}^{2}\right)$ such that $\rho^{0} \geq 0$. Let $\sigma \in C\left(\left[0,+\infty\left[; L^{1}\left(\mathbb{R}^{2}\right)\right) \cap\right.\right.$ $L^{\infty}\left(0,+\infty ; L^{1}\left(\mathbb{R}^{2}\right)\right)$. The problem (4.1) admits a unique weak solution $\rho$, which satisfies $\rho \in L^{\infty}\left(\left[0,+\infty\left[; L^{1}\left(\mathbb{R}^{2}\right)\right) \cap C\left(\left[0,+\infty\left[; L^{1}\left(\mathbb{R}^{2}\right)\right)\right.\right.\right.\right.$. Moreover, if we assume that $\rho^{0} \in L^{\infty}\left(\mathbb{R}^{2}\right)$ is non negative, then we have $\rho \geq 0$ and we can find $C_{0}>0$ such that $0 \leq \rho(t, x) \leq\left\|\rho_{0}\right\|_{L^{\infty}\left(\mathbb{R}^{2}\right)} e^{C_{0} t}$ holds a. $e$.

Proof. Equation (4.1) is a mere transport equation where, owing to Lemma 4.4, the velocity field is regular. As a matter of fact, we can write

$$
\partial_{t} \rho+\operatorname{div}_{x}(\rho \mathcal{U}[\sigma])=0
$$

in the non-conservative form

$$
\partial_{t} \rho+\mathcal{U}[\sigma] \cdot \nabla \rho+\rho \operatorname{div}_{x} \mathcal{U}[\sigma]=0 .
$$

We use the method of characteristics with the function $(t, x) \mapsto \mathcal{U}[\sigma](t, x)$. According to Lemma 4.4, the solutions of

$$
X^{\prime}(t, x)=\mathcal{U}[\sigma](t, X(t, x)), \quad X(0, x)=x
$$


are globally defined. It allows us to express the solution of (4.1) as follows

$$
\rho(t, X(t, x))=\rho_{0}(x) \exp \left(-\int_{0}^{t} \operatorname{div}_{x} \mathcal{U}[\sigma](s, x) \mathrm{d} s\right) .
$$

Clearly, we see that $\rho$ is non negative when $\rho_{0}$ is non negative. Moreover, it satisfies

$$
\|\rho(t, \cdot)\|_{L^{1}\left(\mathbb{R}^{2}\right)} \leq\left\|\rho^{0}\right\|_{L^{1}\left(\mathbb{R}^{2}\right)},
$$

with an equality when the functions are non negative. We can check that $t \mapsto$ $\rho(t, \cdot)$ is continuous with values in $L^{1}\left(\mathbb{R}^{2}\right)$. Finally, to get a $L^{\infty}$ bound for $\rho$, we need to prove that

$$
e^{-\int_{0}^{t} \operatorname{div}_{x} u[\sigma](s, x) \mathrm{d} s}
$$

is bounded. Using Lemma 4.4 again, we obtain

$$
e^{-\int_{0}^{t} \operatorname{div}_{x} \mathcal{U}[\sigma](s, x) \mathrm{d} s} \leq e^{t C_{0}},
$$

with

$$
C_{0}=2 c\left(\mathcal{E}_{1}^{\prime}+\mathcal{E}_{2}^{\prime}\right)\|\sigma\|_{L^{\infty}\left(0, \infty ; L^{1}\left(\mathbb{R}^{2}\right)\right)} e^{4 c \mathcal{E}\|\sigma\|_{L^{\infty}\left(0, \infty ; L^{1}\left(\mathbb{R}^{2}\right)\right)} .}
$$

We conclude that the solution of (4.1) satisfies

$$
|\rho(t, x)| \leq\left\|\rho^{0}\right\|_{L^{\infty}\left(\mathbb{R}^{2}\right)} e^{t C_{0}} .
$$

We shall see that the $L^{\infty}$ estimate equally holds for the solution of the non linear problem; it prevents the solution to blow up in any finite time, and so the equation does not produce delta-Dirac singularities in finite time.

\subsection{Fixed point}

Let $0<T<\infty$. We shall use the space

$$
\begin{aligned}
& X_{T}=\left\{\sigma \in C\left([0, T], L^{1}\left(\mathbb{R}^{2}\right)\right)\right. \text { such that } \\
& \left.\quad \text { for any } 0 \leq t \leq T,\|\sigma(t, \cdot)\|_{L^{1}\left(\mathbb{R}^{2}\right)} \leq\left\|\rho^{0}\right\|_{L^{1}\left(\mathbb{R}^{2}\right)}\right\},
\end{aligned}
$$

which is a Banach space with the norm

$$
\|\sigma\|_{X_{T}}=\sup _{t \in[0, T]}\|\sigma(t, \cdot)\|_{L^{1}\left(\mathbb{R}^{2}\right)} .
$$

We want to prove that the application $\Gamma$ which associates to $\sigma \in X_{T}$ the solution $\rho \in X_{T}$ of Proposition 4.5 is a contraction on $X_{T}$, when $T$ is small enough. The arguments are inspired from the analysis performed in [6] for a non local model of pedestrian flows. In turns out that we need to establish estimates on the flux function

$$
\begin{aligned}
& f_{\sigma}:(0, \infty) \times \mathbb{R}^{2} \times \mathbb{R} \longrightarrow \mathbb{R}^{2} \\
& (t, x, \lambda) \quad \longmapsto \lambda \mathcal{U}[\sigma](t, x) .
\end{aligned}
$$

We bear in mind that this function depends on $\sigma$. To be specific, we are going to establish that $\sigma \mapsto f_{\sigma}$ satisfies certain Lipschitz continuity estimates and that, for any $\sigma \in X_{T}, f_{\sigma}$ satisfies a regularity estimate. In what follows, we shall work with two functions $\sigma_{1}, \sigma_{2}$ in $X_{T}$, and we simply denote $f_{k}$ the flux function associated to $\sigma_{k}$, for $k=1,2$. 
Step 1: Estimate on $\partial_{\lambda}\left(f_{1}-f_{2}\right)$ in $L^{\infty}$.

Lemma 4.6. There exists a constant $C_{1}$, which depends on $c, \mathcal{E},\left\|\rho^{0}\right\|_{L^{1}}$, such that for any $t \in(0, \infty)$ and $x \in \mathbb{R}^{2}$, we have

$$
\left\|\partial_{\lambda}\left(f_{1}-f_{2}\right)(t, x, \cdot)\right\|_{L^{\infty}(\mathbb{R})} \leq C_{1}\left\|\sigma_{2}-\sigma_{1}\right\|_{X_{T}}
$$

Proof. Here, the flux function depends linearly on $\lambda$; we thus have

$$
\left(f_{1}-f_{2}\right)(t, x, \lambda)=\lambda\left(\mathcal{U}\left[\sigma_{1}\right](t, x)-\mathcal{U}\left[\sigma_{2}\right](t, x)\right)
$$

and

$$
\begin{aligned}
\partial_{\lambda}\left(f_{1}-f_{2}\right)(t, x, \lambda)= & \mathcal{U}\left[\sigma_{1}\right](t, x)-\mathcal{U}\left[\sigma_{2}\right](t, x) \\
= & \mu\left[\sigma_{1}\right](t, x) \int_{0}^{2 \pi} \Omega(\theta) e^{-\Psi\left[\sigma_{1}\right](t, x, \theta) / D} \mathrm{~d} \theta \\
& \quad-\mu\left[\sigma_{2}\right](t, x) \int_{0}^{2 \pi} \Omega(\theta) e^{-\Psi\left[\sigma_{2}\right](t, x, \theta) / D} \mathrm{~d} \theta \\
= & \left(\mu\left[\sigma_{1}\right](t, x)-\mu\left[\sigma_{2}\right](t, x)\right) \int_{0}^{2 \pi} \Omega(\theta) e^{-\Psi\left[\sigma_{1}\right](t, x, \theta) / D} \mathrm{~d} \theta \\
& -\mu\left[\sigma_{2}\right](t, x) \int_{0}^{2 \pi} \Omega(\theta)\left(e^{-\Psi\left[\sigma_{2}\right](t, x, \theta) / D}-e^{-\Psi\left[\sigma_{1}\right](t, x, \theta) / D}\right) \mathrm{d} \theta .
\end{aligned}
$$

Now, we observe that

$$
\begin{aligned}
& \Psi\left[\sigma_{1}\right](t, x, \theta)-\Psi\left[\sigma_{2}\right](t, x, \theta) \\
= & \int_{0}^{\theta} \int_{0}^{\ell} \int_{\xi-\beta}^{\xi+\beta} \sin (\xi-\alpha)\left[\left(E * \sigma_{1}\right)(t, x+r \Omega(\alpha))-\left(E * \sigma_{2}\right)(t, x+r \Omega(\alpha))\right] \mathrm{d} \alpha r \mathrm{~d} r \mathrm{~d} \xi \\
= & \int_{0}^{\theta} \int_{0}^{\ell} \int_{\xi-\beta}^{\xi+\beta} \sin (\xi-\alpha)\left(E *\left(\sigma_{1}-\sigma_{2}\right)\right)(t, x+r \Omega(\alpha)) \mathrm{d} \alpha r \mathrm{~d} r \mathrm{~d} \xi \\
= & \Psi\left[\sigma_{1}-\sigma_{2}\right](t, x, \theta)
\end{aligned}
$$

which leads to

$$
\left|\Psi\left[\sigma_{1}\right](t, x, \theta)-\Psi\left[\sigma_{2}\right](t, x, \theta)\right| \leq c D \mathcal{E}\left\|\sigma_{2}-\sigma_{1}\right\|_{X_{T}} .
$$

Next, we make use of the elementary estimate

$$
\text { for any }-A \leq w \leq+A,\left|e^{w}-1\right| \leq e^{A}|w| .
$$

Since the $\sigma_{j}$ 's belong to $X_{T}$, we have

$$
\left\|\sigma_{2}-\sigma_{1}\right\|_{X_{T}} \leq 2\left\|\rho^{0}\right\|_{L^{1}\left(\mathbb{R}^{2}\right)}
$$

Hence, we set $A=2 \mathcal{A}$ with

$$
\mathcal{A}=c \mathcal{E}\left\|\rho^{0}\right\|_{L^{1}\left(\mathbb{R}^{2}\right)}
$$


Coming back to (4.2) yields

$$
\left|e^{\Psi\left[\sigma_{1}\right](t, x, \theta) / D-\Psi\left[\sigma_{2}\right](t, x, \theta) / D}-1\right| \leq e^{2 \mathcal{A}} c \mathcal{E}\left\|\sigma_{2}-\sigma_{1}\right\|_{X_{T}} .
$$

Writing

$$
\begin{aligned}
& e^{-\Psi\left[\sigma_{2}\right](t, x, \theta) / D}-e^{-\Psi\left[\sigma_{1}\right](t, x, \theta) / D} \\
& \quad=e^{-\Psi\left[\sigma_{1}\right](t, x, \theta) / D}\left(e^{\Psi\left[\sigma_{1}\right](t, x, \theta) / D-\Psi\left[\sigma_{2}\right](t, x, \theta) / D}-1\right)
\end{aligned}
$$

we are led to

$$
\left|e^{-\Psi\left[\sigma_{2}\right](t, x, \theta) / D}-e^{-\Psi\left[\sigma_{1}\right](t, x, \theta) / D}\right| \leq e^{3 \mathcal{A}} c \mathcal{E}\left\|\sigma_{2}-\sigma_{1}\right\|_{X_{T}} .
$$

Furthermore, we have

$$
\mu\left[\sigma_{1}\right](t, x)-\mu\left[\sigma_{2}\right](t, x)=\frac{\int_{0}^{2 \pi}\left(e^{-\Psi\left[\sigma_{2}\right](t, x, \theta) / D}-e^{-\Psi\left[\sigma_{1}\right](t, x, \theta) / D}\right) \mathrm{d} \theta}{\int_{0}^{2 \pi} e^{-\Psi\left[\sigma_{1}\right](t, x, \theta) / D} \mathrm{~d} \theta \int_{0}^{2 \pi} e^{-\Psi\left[\sigma_{2}\right](t, x, \theta) / D} \mathrm{~d} \theta},
$$

thus

$$
\begin{aligned}
\left|\mu\left[\sigma_{1}\right](t, x)-\mu\left[\sigma_{2}\right](t, x)\right| \leq & 2 \pi e^{5 \mathcal{A}} c \mathcal{E}\left\|\sigma_{2}-\sigma_{1}\right\|_{X_{T}} \\
& \times \frac{1}{4 \pi^{2}} e^{c \mathcal{E}\left(\left\|\sigma_{1}\right\| X_{T}+\left\|\sigma_{2}\right\|_{X_{T}}\right)} \\
\leq & \frac{c}{2 \pi} e^{7 \mathcal{A}} \mathcal{E}\left\|\sigma_{2}-\sigma_{1}\right\|_{X_{T}} .
\end{aligned}
$$

Therefore we get

$$
\left\|\partial_{\lambda}\left(f_{1}-f_{2}\right)(t, x, \lambda)\right\| \leq c \mathcal{E} e^{4 \mathcal{A}}\left(1+e^{4 \mathcal{A}}\right)\left\|\sigma_{2}-\sigma_{1}\right\|_{X_{T}}
$$

which ends the proof.

Step 2: Estimate on $\operatorname{div}_{x}\left(f_{2}-f_{1}\right)$ in $L^{1}$.

Lemma 4.7. There exists a constant $C_{2}$, which depends on $c, \mathcal{E},\left\|\rho^{0}\right\|_{L^{1}}$, such that for any $0<R<\infty$, we have

$$
\begin{aligned}
\iint_{\mathbb{R}^{2}} \sup _{0 \leq \lambda \leq R} & \left\|\operatorname{div}_{x}\left(f_{2}-f_{1}\right)(t, x, \lambda)\right\| \mathrm{d} x \\
\leq & \left(\left\|\partial_{x_{1}} E\right\|_{L^{1}\left(\mathbb{R}^{2}\right)}+\left\|\partial_{x_{2}} E\right\|_{L^{1}\left(\mathbb{R}^{2}\right)}\right) C_{2} R\left\|\sigma_{2}-\sigma_{1}\right\|_{X_{T}} .
\end{aligned}
$$

Proof. We have

$$
\operatorname{div}_{x}\left(f_{2}-f_{1}\right)(t, x, \lambda)=\lambda \operatorname{div}_{x}\left(\mathcal{U}\left[\sigma_{2}\right]-\mathcal{U}\left[\sigma_{1}\right]\right)(t, x) .
$$


We compute $\operatorname{div}_{x}\left(\mathcal{U}\left[\sigma_{2}\right]-\mathcal{U}\left[\sigma_{1}\right]\right)$ by using (4.12) and we reorganize terms as follows

$$
\begin{aligned}
& \operatorname{div}_{x}\left(\mathcal{U}\left[\sigma_{2}\right]-\mathcal{U}\left[\sigma_{1}\right]\right)(t, x) \\
= & \left.\partial_{x_{1}} \mu\left[\sigma_{2}\right](t, x)-\partial_{x_{1}} \mu\left[\sigma_{1}\right](t, x)\right) \int_{0}^{2 \pi} \cos (\theta) e^{-\Psi\left[\sigma_{2}\right](t, x, \theta) / D} \mathrm{~d} \theta \\
+ & \partial_{x_{1}} \mu\left[\sigma_{1}\right](t, x) \int_{0}^{2 \pi} \cos (\theta)\left(e^{-\Psi\left[\sigma_{2}\right](t, x, \theta) / D}-e^{-\Psi\left[\sigma_{1}\right](t, x, \theta) / D}\right) \mathrm{d} \theta \\
+ & \left.\left(\partial_{x_{2}} \mu\left[\sigma_{2}\right](t, x)-\partial_{x_{2}} \mu\left[\sigma_{1}\right](t, x)\right)\right) \int_{0}^{2 \pi} \sin (\theta) e^{-\Psi\left[\sigma_{2}\right](t, x, \theta) / D} \mathrm{~d} \theta \\
+ & \partial_{x_{2}} \mu\left[\sigma_{1}\right](t, x) \int_{0}^{2 \pi} \sin (\theta)\left(e^{-\Psi\left[\sigma_{2}\right](t, x, \theta) / D}-e^{-\Psi\left[\sigma_{1}\right](t, x, \theta) / D}\right) \mathrm{d} \theta \\
+ & \frac{1}{D}\left(\mu\left[\sigma_{2}\right](t, x)-\mu\left[\sigma_{1}\right](t, x)\right) \int_{0}^{2 \pi} \cos (\theta) \partial_{x_{1}} \Psi\left[\sigma_{2}\right](t, x, \theta) e^{-\Psi\left[\sigma_{2}\right](t, x, \theta) / D} \mathrm{~d} \theta \\
+ & \frac{1}{D} \mu\left[\sigma_{1}\right](t, x) \int_{0}^{2 \pi} \cos (\theta) \\
& \times\left(\partial_{x_{1}} \Psi\left[\sigma_{1}\right](t, x, \theta) e^{-\Psi\left[\sigma_{1}\right](t, x, \theta) / D}-\partial_{x_{1}} \Psi\left[\sigma_{2}\right](t, x, \theta) e^{-\Psi\left[\sigma_{2}\right](t, x, \theta) / D}\right) \mathrm{d} \theta \\
+ & \frac{1}{D}\left(\mu\left[\sigma_{2}\right](t, x)-\mu\left[\sigma_{1}\right](t, x)\right) \int_{0}^{2 \pi} \sin (\theta) \partial_{x_{2}} \Psi\left[\sigma_{2}\right](t, x, \theta) e^{-\Psi\left[\sigma_{2}\right](t, x, \theta) / D} \mathrm{~d} \theta \\
+ & \frac{1}{D} \mu\left[\sigma_{1}\right](t, x) \int_{0}^{2 \pi} \sin (\theta) \\
& \times\left(\partial_{x_{2}} \Psi\left[\sigma_{1}\right](t, x, \theta) e^{-\Psi\left[\sigma_{1}\right](t, x, \theta) / D}-\partial_{x_{2}} \Psi\left[\sigma_{2}\right](t, x, \theta) e^{-\Psi\left[\sigma_{2}\right](t, x, \theta) / D}\right) \mathrm{d} \theta .
\end{aligned}
$$

Since $\Psi$ depends linearly on $\sigma$, we get

$$
\begin{aligned}
& \partial_{x_{k}} \Psi\left[\sigma_{1}\right](t, x, \theta) e^{-\Psi\left[\sigma_{1}\right](t, x, \theta) / D}-\partial_{x_{k}} \Psi\left[\sigma_{2}\right](t, x, \theta) e^{-\Psi\left[\sigma_{2}\right](t, x, \theta) / D} \\
= & \partial_{x_{k}} \Psi\left[\sigma_{1}-\sigma_{2}\right](t, x, \theta) e^{-\Psi\left[\sigma_{1}\right](t, x, \theta) / D} \\
& +\partial_{x_{k}} \Psi\left[\sigma_{2}\right](t, x, \theta)\left(e^{-\Psi\left[\sigma_{1}\right](t, x, \theta) / D}-e^{-\Psi\left[\sigma_{2}\right](t, x, \theta) / D}\right) .
\end{aligned}
$$

Going back to (4.6), together with (4.13), we arrive at

$$
\begin{aligned}
& \iint_{\mathbb{R}^{2}}\left|\partial_{x_{k}} \Psi\left[\sigma_{1}\right](t, x, \theta) e^{-\Psi\left[\sigma_{1}\right](t, x, \theta) / D}-\partial_{x_{k}} \Psi\left[\sigma_{2}\right](t, x, \theta) e^{-\Psi\left[\sigma_{2}\right](t, x, \theta) / D}\right| \mathrm{d} x \\
\leq & c D e^{\mathcal{A}}\left\|\partial_{x_{k}} E\right\|_{L^{1}\left(\mathbb{R}^{2}\right)}\left\|\sigma_{2}-\sigma_{1}\right\|_{X_{T}}+c^{2} D \mathcal{E} e^{3 \mathcal{A}}\left\|\partial_{x_{k}} E\right\|_{L^{1}\left(\mathbb{R}^{2}\right)}\left\|\sigma_{2}-\sigma_{1}\right\|_{X_{T}} \times\left\|\sigma_{2}\right\|_{X_{T}} .
\end{aligned}
$$

Furthermore, we have

$$
\begin{aligned}
& \partial_{x_{k}} \mu\left[\sigma_{2}\right](t, x)-\partial_{x_{k}} \mu\left[\sigma_{1}\right](t, x) \\
= & \frac{1}{D} \int_{0}^{2 \pi} \partial_{x_{k}} \Psi\left[\sigma_{2}\right](t, x, \theta) e^{-\Psi\left[\sigma_{2}\right](t, x, \theta) / D} \mathrm{~d} \theta\left|\mu\left[\sigma_{2}\right](t, x)\right|^{2} \\
& -\frac{1}{D} \int_{0}^{2 \pi} \partial_{x_{k}} \Psi\left[\sigma_{1}\right](t, x, \theta) e^{-\Psi\left[\sigma_{1}\right](t, x, \theta) / D} \mathrm{~d} \theta\left|\mu\left[\sigma_{1}\right](t, x)\right|^{2}
\end{aligned}
$$




$$
\begin{aligned}
= & \frac{1}{D} \int_{0}^{2 \pi}\left(\partial_{x_{k}} \Psi\left[\sigma_{2}\right](t, x, \theta) e^{-\Psi\left[\sigma_{2}\right](t, x, \theta) / D}\right. \\
& \left.-\partial_{x_{k}} \Psi\left[\sigma_{1}\right](t, x, \theta) e^{-\Psi\left[\sigma_{1}\right](t, x, \theta) / D}\right) \mathrm{d} \theta\left|\mu\left[\sigma_{2}\right](t, x)\right|^{2} \\
& +\frac{1}{D} \int_{0}^{2 \pi} \partial_{x_{k}} \Psi\left[\sigma_{1}\right](t, x, \theta) e^{-\Psi\left[\sigma_{1}\right](t, x, \theta) / D} \mathrm{~d} \theta\left(\left|\mu\left[\sigma_{2}\right](t, x)\right|^{2}-\left|\mu\left[\sigma_{1}\right](t, x)\right|^{2}\right) .
\end{aligned}
$$

Similarly, the $L^{1}$ norm of this quantity can be dominated by

$$
C_{2}\left\|\partial_{x_{k}} E\right\|_{L^{1}\left(\mathbb{R}^{2}\right)}\left\|\sigma_{1}-\sigma_{2}\right\|_{X_{T}}
$$

with a certain constant $C_{2}$ that depends on $c, \mathcal{E},\left\|\rho^{0}\right\|_{L^{1}}$. This allows us to conclude, bearing in mind that

$$
\begin{aligned}
\iint_{\mathbb{R}^{2}} \sup _{0 \leq \lambda \leq R}\left\|\operatorname{div}_{x}\left(f_{1}-f_{2}\right)(t, x, \lambda)\right\| \mathrm{d} x \mathrm{~d} t \\
\leq R \iint_{\mathbb{R}^{2}}\left\|\operatorname{div}_{x}\left(\mathcal{U}\left[\sigma_{1}\right]-\mathcal{U}\left[\sigma_{2}\right]\right)(t, x)\right\| \mathrm{d} x \mathrm{~d} t .
\end{aligned}
$$

Step 3: Estimate on $\nabla \operatorname{div}_{x} f$ in $L^{1}$.

Lemma 4.8. There exists constants $C_{3}$ and $C_{4}$, depending only on $c, D, \mathcal{E},\left\|\rho^{0}\right\|_{L^{1}}$, on $\mathcal{E}_{1}^{\prime}, \mathcal{E}_{2}^{\prime}$, and on the $L^{1}$ norm of the first and second derivatives of $E$, such that, for any $0<R<\infty$

$$
\iint_{\mathbb{R}^{2}} \sup _{0 \leq \lambda \leq R}\left\|\nabla \operatorname{div}_{x} f(t, x, \lambda)\right\| \mathrm{d} x \leq R\left(C_{3}+C_{4}\left\|\rho^{0}\right\|_{L^{1}\left(\mathbb{R}^{2}\right)}\right)\left\|\rho^{0}\right\|_{L^{1}\left(\mathbb{R}^{2}\right)}
$$

Proof. We have

$$
\nabla \operatorname{div}_{x} f(t, x, \lambda)=\lambda\left(\partial_{x_{1}} \operatorname{div}_{x} \mathcal{U}[\sigma], \partial_{x_{2}} \operatorname{div}_{x} \mathcal{U}[\sigma]\right)
$$

so that the difficulty reduces to discuss the $L^{1}$ norm of

$$
\begin{aligned}
& \partial_{x_{k}} \operatorname{div}_{x} \mathcal{U}[\sigma](t, x) \\
& =\partial_{x_{k} x_{1}}^{2} \mu[\sigma](x) \int_{0}^{2 \pi} \cos (\theta) e^{-\Psi[\sigma](x, \theta) / D} \mathrm{~d} \theta \\
& \quad-\frac{1}{D} \partial_{x_{1}} \mu[\sigma](x) \int_{0}^{2 \pi} \cos (\theta) \partial_{x_{k}} \Psi[\sigma](x, \theta) e^{-\Psi[\sigma](x, \theta) / D} \mathrm{~d} \theta \\
& \quad-\frac{1}{D} \partial_{x_{k}} \mu[\sigma](x) \int_{0}^{2 \pi} \cos (\theta) \partial_{x_{1}} \Psi[\sigma](x, \theta) e^{-\Psi[\sigma](x, \theta) / D} \mathrm{~d} \theta \\
& \quad-\frac{1}{D} \mu[\sigma](x) \int_{0}^{2 \pi} \cos (\theta) \partial_{x_{k} x_{1}}^{2} \Psi[\sigma](x, \theta) e^{-\Psi[\sigma](x, \theta) / D} \mathrm{~d} \theta \\
& +\frac{1}{D^{2}} \mu[\sigma](x) \int_{0}^{2 \pi} \cos (\theta) \partial_{x_{1}} \Psi[\sigma](x, \theta) \partial_{x_{k}} \Psi[\sigma](x, \theta) e^{-\Psi[\sigma](x, \theta) / D} \mathrm{~d} \theta
\end{aligned}
$$




$$
\begin{aligned}
& +\partial_{x_{k} x_{2}}^{2} \mu[\sigma](x) \int_{0}^{2 \pi} \sin (\theta) e^{-\Psi[\sigma](x, \theta) / D} \mathrm{~d} \theta \\
& -\frac{1}{D} \partial_{x_{2}} \mu[\sigma](x) \int_{0}^{2 \pi} \sin (\theta) \partial_{x_{k}} \Psi[\sigma](x, \theta) e^{-\Psi[\sigma](x, \theta) / D} \mathrm{~d} \theta \\
& -\frac{1}{D} \partial_{x_{k}} \mu[\sigma](x) \int_{0}^{2 \pi} \sin (\theta) \partial_{x_{2}} \Psi[\sigma](x, \theta) e^{-\Psi[\sigma](x, \theta) / D} \mathrm{~d} \theta \\
& -\frac{1}{D} \mu[\sigma](x) \int_{0}^{2 \pi} \sin (\theta) \partial_{x_{k} x_{2}}^{2} \Psi[\sigma](x, \theta) e^{-\Psi[\sigma](x, \theta) / D} \mathrm{~d} \theta \\
& +\frac{1}{D^{2}} \mu[\sigma](x) \int_{0}^{2 \pi} \sin (\theta) \partial_{x_{2}} \Psi[\sigma](x, \theta) \partial_{x_{k}} \Psi[\sigma](x, \theta) e^{-\Psi[\sigma](x, \theta) / D} \mathrm{~d} \theta .
\end{aligned}
$$

We use Lemma 4.2 and 4.3 to conclude. We use one $L^{1}$ bound and $L^{\infty}$ bounds for every product. The $L^{1}$ norm are $\partial_{x_{k} x_{l}}^{2} \mu[\sigma], \partial_{x_{k}} \mu[\sigma]$, for the first, second, third, sixth, seventh, eighth terms and $\partial_{x_{k} x_{l}}^{2} \Psi[\sigma], \partial_{x_{k}} \Psi[\sigma]$ for the forth, fifth, nineth and tenth terms. We observe that $C_{3}$ depends on the $L^{1}$ norm of the second derivatives of $E$, while $C_{4}$ depends on the products $\mathcal{E}_{k}^{\prime}\left\|\partial_{x_{j}} E\right\|_{L^{1}\left(\mathbb{R}^{2}\right)}$.

Theorem 4.9. Let $\rho^{0} \in L^{1}\left(\mathbb{R}^{2}\right) \cap L^{\infty} \cap B V\left(\mathbb{R}^{2}\right)$ such that $\rho^{0} \geq 0$. There exists $T_{1}>0$ such that there exists a unique weak entropy solution on $\left[0, T_{1}\right]$ to

$$
\left\{\begin{aligned}
\partial_{t} \rho+\operatorname{div}_{x}(\rho \mathcal{U}[\rho]) & =0 \\
\rho(0, x) & =\rho^{0}(x) .
\end{aligned}\right.
$$

Proof. Let us start by introducing a few notations. Let

$$
W_{2}=\int_{0}^{\pi / 2}|\cos (\theta)|^{2} \mathrm{~d} \theta
$$

We also set

$$
\kappa=\left\|\partial_{\lambda} \operatorname{div}_{x}\left(f_{1}-f_{2}\right)\right\|_{L^{\infty}}, \quad \kappa_{0}=5\left\|\nabla \partial_{\lambda} f\right\|_{L^{\infty}} .
$$

We have

$$
\partial_{x} \operatorname{div}_{x}\left(f_{1}-f_{2}\right)=\operatorname{div}_{x}\left(\mathcal{U}\left[\sigma_{2}\right]-\mathcal{U}\left[\sigma_{1}\right]\right)
$$

Reproducing the analysis in Lemma 4.7, we obtain

$$
\kappa \leq C_{2}\left(\mathcal{E}_{1}^{\prime}+\mathcal{E}_{2}^{\prime}\right) e^{8 \mathcal{A}}\left\|\sigma_{2}-\sigma_{1}\right\|_{X_{T}} \leq 2 C_{2}\left(\mathcal{E}_{1}^{\prime}+\mathcal{E}_{2}^{\prime}\right) e^{8 \mathcal{A}}\left\|\rho^{0}\right\|_{L^{1}\left(\mathbb{R}^{2}\right)} .
$$

Moreover, observing that

$$
\nabla \partial_{\lambda} f=\nabla \mathcal{U}[\sigma]
$$

we obtain

$$
\kappa_{0} \leq 5 c\left(\mathcal{E}_{1}^{\prime}+\mathcal{E}_{2}^{\prime}\right)\|\sigma\|_{X_{T}} e^{4 c \mathcal{E}\|\sigma\|_{X_{T}}} \leq 5 c\left(\mathcal{E}_{1}^{\prime}+\varepsilon_{2}^{\prime}\right)\left\|\rho^{0}\right\|_{L^{1}\left(\mathbb{R}^{2}\right)} e^{4 \mathcal{A}} .
$$

Setting $\kappa_{1}=\max \left(\kappa, \kappa_{0}\right)$, we finally observe that

$$
\frac{e^{\kappa_{0} s}-e^{\kappa s}}{\kappa_{0}-\kappa} \leq s e^{\kappa_{1} s}
$$


Let us denote

$$
R(T)=\left\|\rho^{0}\right\|_{L^{\infty}\left(\mathbb{R}^{2}\right)} e^{T C_{0}},
$$

which is the $L^{\infty}$ bound obtained in Proposition 4.5. Finally, let

$$
K(T)=e^{\kappa_{1} T}\left[C_{1} \operatorname{TV}\left(\rho^{0}\right)+R(T)\left(C_{5} T+C_{6}\right)\right]
$$

with

$$
C_{5}=2 W_{2} C_{1}\left(C_{3}+C_{4}\left\|\rho^{0}\right\|_{L^{1}\left(\mathbb{R}^{2}\right)}\right)\left\|\rho^{0}\right\|_{L^{\infty}\left(\mathbb{R}^{2}\right)}
$$

and

$$
C_{6}=C_{2}\left(\left\|\partial_{x_{1}} E\right\|_{L^{1}\left(\mathbb{R}^{2}\right)}+\left\|\partial_{x_{2}} E\right\|_{L^{1}\left(\mathbb{R}^{2}\right)}\right)\left\|\rho^{0}\right\|_{L^{\infty}\left(\mathbb{R}^{2}\right)} .
$$

The function $t \mapsto t K(t)$ is continuous and satisfies

$$
\left.t K(t)\right|_{t=0}=0, \quad \lim _{t \rightarrow \infty} t K(t)=+\infty .
$$

Hence, there exists $T_{1}>0$ such that

$$
T_{1} K\left(T_{1}\right)=1 / 2 \text { and } 0<t K(t)<1 / 2 \text { for any } t \in\left(0, T_{1}\right) .
$$

Using [19, Theorem 2.5], we have for $t \in\left[0, T_{1}\right]$,

$$
\begin{aligned}
& \iint_{\mathbb{R}^{2}}\left|\rho_{1}(t, x)-\rho_{2}(t, x)\right| \mathrm{d} x \\
\leq & \frac{e^{\kappa_{0} t}-e^{\kappa t}}{\kappa_{0}-\kappa} \mathrm{TV}\left(\rho^{0}\right)\left\|\partial_{\lambda}\left(f_{1}-f_{2}\right)\right\|_{L^{\infty}} \\
+ & 2 W_{2}\left(\int_{0}^{t} \frac{e^{\kappa_{0}(t-s)}-e^{\kappa(t-s)}}{\kappa_{0}-\kappa}\right. \\
& \left.\times \iint_{\mathbb{R}^{2}} \sup _{0 \leq \lambda \leq R(T)}\left\|\nabla \operatorname{div}_{x} f_{1}(s, x, \lambda)\right\| \mathrm{d} x \mathrm{~d} s\right)\left\|\partial_{\lambda}\left(f_{1}-f_{2}\right)\right\|_{L^{\infty}} \\
+ & \int_{0}^{t} e^{\kappa(t-s)} \iint_{\mathbb{R}^{2}} \sup _{0 \leq \lambda \leq R(T)}\left\|\operatorname{div}_{x}\left(f_{2}-f_{1}\right)(s, x, \lambda)\right\| \mathrm{d} x \mathrm{~d} s .
\end{aligned}
$$

Owing to Lemma 4.6, 4.7 and 4.8 we get, for $t \in\left[0, T_{1}\right]$,

$$
\begin{aligned}
& \iint_{\mathbb{R}^{2}}\left|\rho_{1}(t, x)-\rho_{2}(t, x)\right| \mathrm{d} x \\
\leq & t e^{\kappa_{1} t} \operatorname{TV}\left(\rho^{0}\right) C_{1}\left\|\sigma_{2}-\sigma_{1}\right\|_{X_{T}} \\
& +2 W_{2}\left(\int_{0}^{t}(t-s) e^{\kappa_{1}(t-s)} R\left(T_{1}\right)\left(C_{3}+C_{4}\left\|\rho^{0}\right\|_{L^{1}\left(\mathbb{R}^{2}\right)}\right)\left\|\rho^{0}\right\|_{L^{1}\left(\mathbb{R}^{2}\right)} \mathrm{d} s\right) C_{1}\left\|\sigma_{2}-\sigma_{1}\right\|_{X_{T}} \\
& +\int_{0}^{t} e^{\kappa(t-s)} R\left(T_{1}\right) C_{2}\left(\left\|\partial_{x_{1}} E\right\|_{L^{1}\left(\mathbb{R}^{2}\right)}+\left\|\partial_{x_{2}} E\right\|_{L^{1}\left(\mathbb{R}^{2}\right)}\right)\left\|\sigma_{2}-\sigma_{1}\right\|_{X_{T}} \mathrm{~d} s \\
\leq & t K\left(T_{1}\right)\left\|\sigma_{2}-\sigma_{1}\right\|_{X_{T_{1}}} \\
\leq & \frac{1}{2}\left\|\sigma_{2}-\sigma_{1}\right\|_{X_{T_{1}}} .
\end{aligned}
$$

Therefore, the mapping $\sigma \mapsto \rho$ is a contraction on $X_{T_{1}}$ and admits a unique fixed point. It gives a solution to the problem. 


\section{4 $B V$ estimates and infinite lifespan}

Proposition 4.10. There exists a constant $C_{7}$, depending only on $c, D, \mathcal{E},\left\|\rho^{0}\right\|_{L^{1}}$, $\left\|\rho^{0}\right\|_{L^{\infty}}, \mathcal{E}_{1}^{\prime}, \mathcal{E}_{2}^{\prime}$, and on the $L^{1}$ norm of the first and second derivatives of $E$, such that the solution of Theorem 4.9 satisfies

$$
\operatorname{TV}(\rho(t)) \leq\left(\operatorname{TV}\left(\rho^{0}\right)+C_{7} t e^{T_{1} C_{0}}\right) e^{\kappa_{0} t},
$$

for any $t \in\left[0, T_{1}\right]$.

Proof. We keep the same notation as in the proof of Theorem 4.9. Using [19, Theorem 2.2], we have

$$
\begin{aligned}
\operatorname{TV}(\rho(t)) \leq & \operatorname{TV}\left(\rho^{0}\right) e^{\kappa_{0} t} \\
& +2 W_{2} \int_{0}^{t} e^{\kappa_{0}(t-s)} \iint_{\mathbb{R}^{2}} \sup _{0 \leq \lambda \leq R(t)}\left\|\nabla \operatorname{div}_{x} f(s, x, \lambda)\right\| \mathrm{d} x \mathrm{~d} s .
\end{aligned}
$$

By using Lemma 4.8, we are led to

$$
\operatorname{TV}(\rho(t)) \leq \operatorname{TV}\left(\rho^{0}\right) e^{\kappa_{0} t}+2 W_{2} t e^{\kappa_{0} t} R\left(T_{1}\right)\left(C_{3}+C_{4}\left\|\rho^{0}\right\|_{L^{1}\left(\mathbb{R}^{2}\right)}\right)\left\|\rho^{0}\right\|_{L^{1}\left(\mathbb{R}^{2}\right)} .
$$

We get the announced property with

$$
C_{7}=2 W_{2}\left\|\rho^{0}\right\|_{L^{\infty}\left(\mathbb{R}^{2}\right)}\left(C_{3}+C_{4}\left\|\rho^{0}\right\|_{L^{1}\left(\mathbb{R}^{2}\right)}\right)\left\|\rho^{0}\right\|_{L^{1}\left(\mathbb{R}^{2}\right)}^{2} .
$$

Theorem 4.11. Let $\rho^{0} \in L^{1}\left(\mathbb{R}^{2}\right) \cap L^{\infty} \cap B V\left(\mathbb{R}^{2}\right)$ such that $\rho^{0} \geq 0$. There exists a weak entropy solution on $\mathbb{R}^{+}$to

$$
\left\{\begin{aligned}
\partial_{t} \rho+\operatorname{div}_{x}(\rho \mathcal{U}[\rho]) & =0 \\
\rho(0, x) & =\rho^{0}(x) .
\end{aligned}\right.
$$

Proof. We construct now the solution on $\left[T_{n}, T_{n+1}\right]$ starting at the left end time from the initial data $\rho\left(T_{n}\right)$. Since the $L^{1}$ norm is preserved, terms with $\left\|\rho^{0}\right\|_{L^{1}\left(\mathbb{R}^{2}\right)}$ are unchanged. The estimate on $\operatorname{TV}(\rho(t))$ is not that simple.

We set $T_{0}=0$ and we assume we have constructed the solution on $\bigcup_{k=0}^{n-1}\left[T_{k}, T_{k+1}\right]$. Using Proposition 4.10, going from 0 to $t \in\left[0, T_{n}\right]$, we have

$$
\operatorname{TV}(\rho(t)) \leq\left(\operatorname{TV}\left(\rho^{0}\right)+C_{7} t e^{T_{n} C_{0}}\right) e^{\kappa_{0} t} .
$$

We set

$$
\begin{aligned}
K_{n}(T)= & e^{\kappa_{1}\left(T-T_{n}\right)}\left(\mathrm{TV}\left(\rho^{0}\right)+C_{7} T_{n} e^{T_{n} C_{0}}\right) e^{\kappa_{0} T_{n}} C_{1} \\
& +\left(T-T_{n}\right) e^{\kappa_{1}\left(T-T_{n}\right)} e^{T_{n+1} C_{0}} C_{5}+e^{\kappa\left(T-T_{n}\right)} e^{T C_{0}} C_{6} .
\end{aligned}
$$

We observe that

$$
\left.\left(T-T_{n}\right) K_{n}(T)\right|_{T=T_{n}}=0, \quad \lim _{T \rightarrow \infty}\left(T-T_{n}\right) K_{n}(T)=+\infty
$$

so that, by continuity, we can find $T_{n+1}>T_{n}$ such that

$$
\left(T_{n+1}-T_{n}\right) K_{n}\left(T_{n+1}\right)=\frac{1}{2}
$$


Having at hand this definition, and using the same notations as above, we are going to show that $\Gamma$ is still a contraction which allows us to construct a solution to the non linear problem on $\left[T_{n}, T_{n+1}\right]$.

Using [19] and starting from $T_{n}$, we have for $t \in\left[T_{n}, T_{n+1}\right]$,

$$
\begin{aligned}
& \iint_{\mathbb{R}^{2}}\left|\rho_{1}(t, x)-\rho_{2}(t, x)\right| \mathrm{d} x \\
\leq & \left(\left(t-T_{n}\right) e^{\kappa_{1}\left(t-T_{n}\right)} \mathrm{TV}\left(\rho\left(T_{n}\right)\right) C_{1}+\int_{T_{n}}^{t}(t-s) e^{\kappa_{1}(t-s)} e^{T_{n+1} C_{0}} C_{5} \mathrm{~d} s\right. \\
& \left.\quad+\int_{T_{n}}^{t} e^{\kappa(t-s)} e^{T_{n+1} C_{0}} C_{6} \mathrm{~d} s\right)\left\|\sigma_{2}-\sigma_{1}\right\|_{X_{T_{n+1}}} \\
\leq \quad & \left(t-T_{n}\right)\left(e^{\kappa_{1}\left(t-T_{n}\right)} \mathrm{TV}\left(\rho\left(T_{n}\right)\right) C_{1}+\left(T_{n+1}-T_{n}\right) e^{\kappa_{1}\left(t-T_{n}\right)} e^{T_{n+1} C_{0}} C_{5}\right. \\
& \left.\quad+e^{\kappa\left(t-T_{n}\right)} e^{T_{n+1} C_{0}} C_{6}\right)\left\|\sigma_{2}-\sigma_{1}\right\|_{X_{T_{n+1}}} \\
\leq \quad & \left(t-T_{n}\right)\left(e^{\kappa_{1}\left(T_{n+1}-T_{n}\right)}\left(\mathrm{TV}\left(\rho^{0}\right)+C_{7} T_{n} e^{T_{n} C_{0}}\right) e^{\kappa_{0} T_{n}} C_{1}\right. \\
& \left.+\left(T_{n+1}-T_{n}\right) e^{\kappa_{1}\left(T_{n+1}-T_{n}\right)} e^{T_{n+1} C_{0}} C_{5}+e^{\kappa\left(T_{n+1}-T_{n}\right)} e^{T_{n+1} C_{0}} C_{6}\right) \\
\leq & \left(t-T_{n}\right) K_{n}\left(T_{n+1}\right)\left\|\sigma_{2}-\sigma_{1}\right\|_{X_{T_{n+1}}} \|_{X_{T_{n+1}}} \\
\leq & \frac{1}{2}\left\|\sigma_{2}-\sigma_{1}\right\| X_{T_{n+1}} \cdot
\end{aligned}
$$

We have a contraction and we get a solution on $\left[T_{n}, T_{n+1}\right]$ which leads to a solution on $\bigcup_{k=0}^{n}\left[T_{k}, T_{k+1}\right]$. Then by induction, we have obtained a solution on $\bigcup_{k \in \mathbb{N}}\left[T_{k}, T_{k+1}\right]$. We end the proof by showing that $T_{n} \rightarrow+\infty$ when $n \rightarrow+\infty$. By construction the sequence $\left(T_{n}\right)_{n \in \mathbb{N}}$ is strictly increasing. Let us assume that this sequence is bounded, so that $T_{n} \rightarrow T^{*}<+\infty$ as $n \rightarrow \infty$. By the definition of $K_{n}$, we see that the sequence $\left(K_{n}\left(T_{n+1}\right)\right)_{n \in \mathbb{N}}$ is bounded too. Furthermore, $T_{n+1}-T_{n} \rightarrow 0$ so that the left hand side in (4.17) tends to 0 as $n \rightarrow \infty$, a contradiction. We conclude that $\left(T_{n}\right)_{n \in \mathbb{N}}$ is not bounded and $T_{n} \rightarrow+\infty$.

\section{Acknowledgements}

We acknowledge support form the Brazilian-French Network in Mathematics, which has made possible a visit in Nice where a large part of this work was done. P.A. was also partially supported by FAPERJ grant "Jovem Cientista do Nosso Estado" no. 202.867/2015. 


\section{A Proof of the technical lemmas}

\section{Proof of Lemma 4.2}

We start by noticing that

$$
|(E * \sigma)(x)| \leq\|E\|_{L^{\infty}\left(\mathbb{R}^{2}\right)}\|\sigma\|_{L^{1}\left(\mathbb{R}^{2}\right)}=\mathcal{E}\|\sigma\|_{L^{1}\left(\mathbb{R}^{2}\right)},
$$

which implies

$$
\begin{aligned}
|\Psi[\sigma](x, \theta)| & \leq \int_{0}^{\theta} \int_{0}^{\ell} \int_{\xi-\beta}^{\xi+\beta}|(E * \sigma)(x+r \Omega(\alpha))| \mathrm{d} \alpha r \mathrm{~d} r \mathrm{~d} \xi \\
& \leq \ell \int_{0}^{\theta} \int_{0}^{\ell} \int_{\xi-\beta}^{\xi+\beta} \mathcal{E}\|\sigma\|_{L^{1}\left(\mathbb{R}^{2}\right)} \mathrm{d} \alpha \mathrm{d} r \mathrm{~d} \xi \\
& \leq(2 \pi \ell)^{2} \mathcal{E}\|\sigma\|_{L^{1}\left(\mathbb{R}^{2}\right)}
\end{aligned}
$$

It follows that

$$
e^{-c \mathcal{E}\|\sigma\|_{L^{1}\left(\mathbb{R}^{2}\right)}} \leq e^{-\Psi[\sigma](x, \theta) / D} \leq e^{c \mathcal{E}\|\sigma\|_{L^{1}\left(\mathbb{R}^{2}\right)}} .
$$

We also obtain

$$
|\mu[\sigma](x)| \leq \frac{1}{2 \pi} e^{c \mathcal{E}\|\sigma\|_{L^{1}\left(\mathbb{R}^{2}\right)}} .
$$

Next, we have

$$
\partial_{x_{k}} \Psi[\sigma](x, \theta)=\int_{0}^{\theta} \int_{0}^{\ell} \int_{\xi-\beta}^{\xi+\beta} \sin (\xi-\alpha)\left(\partial_{x_{k}} E * \sigma\right)(x+r \Omega(\alpha)) \mathrm{d} \alpha r \mathrm{~d} r \mathrm{~d} \xi
$$

and

$$
\partial_{x_{k}} \mu[\sigma](x)=\frac{|\mu[\sigma](x)|^{2}}{D} \int_{0}^{2 \pi} \partial_{x_{k}} \Psi[\sigma](x, \theta) e^{-\Psi[\sigma](x, \theta) / D} \mathrm{~d} \theta .
$$

We get

$$
\left|\partial_{x_{k}} \Psi[\sigma](x, \theta)\right| \leq(2 \pi \ell)^{2} \mathcal{E}_{k}^{\prime}\|\sigma\|_{L^{1}\left(\mathbb{R}^{2}\right)}
$$

and

$$
\begin{aligned}
\left|\partial_{x_{k}} \mu[\sigma](x)\right| & \leq 2 \pi c \mathcal{E}_{k}^{\prime}\|\sigma\|_{L^{1}\left(\mathbb{R}^{2}\right)} e^{c \mathcal{E}\|\sigma\|_{L^{1}\left(\mathbb{R}^{2}\right)}}|\mu[\sigma](x)|^{2} \\
& \leq \frac{c}{2 \pi} \mathcal{E}_{k}^{\prime}\|\sigma\|_{L^{1}\left(\mathbb{R}^{2}\right)} e^{3 c \mathcal{E}\|\sigma\|_{L^{1}\left(\mathbb{R}^{2}\right)}} .
\end{aligned}
$$

Finally, we have

$$
\partial_{x_{k} x_{l}}^{2} \Psi[\sigma](x, \theta)=\int_{0}^{\theta} \int_{0}^{\ell} \int_{\xi-\beta}^{\xi+\beta} \sin (\xi-\alpha)\left(\partial_{x_{k} x_{l}}^{2} E * \sigma\right)(x+r \Omega(\alpha)) \mathrm{d} \alpha r \mathrm{~d} r \mathrm{~d} \xi
$$


and

$$
\begin{aligned}
\partial_{x_{k} x_{l}}^{2} \mu[\sigma](x)= & \frac{|\mu[\sigma](x)|^{2}}{D} \int_{0}^{2 \pi} \partial_{x_{k} x_{l}}^{2} \Psi[\sigma](x, \theta) e^{-\Psi[\sigma](x, \theta) / D} \mathrm{~d} \theta \\
& -\frac{|\mu[\sigma](x)|^{2}}{D^{2}} \int_{0}^{2 \pi} \partial_{x_{k}} \Psi[\sigma](x, \theta) \partial_{x_{l}} \Psi[\sigma](x, \theta) e^{-\Psi[\sigma](x, \theta) / D} \mathrm{~d} \theta \\
& +\frac{2}{D} \int_{0}^{2 \pi} \partial_{x_{l}} \Psi[\sigma](x, \theta) e^{-\Psi[\sigma](x, \theta) / D} \mathrm{~d} \theta \times \mu[\sigma](x) \partial_{x_{k}} \mu[\sigma](x) .
\end{aligned}
$$

Hence, we get

$$
\left|\partial_{x_{k} x_{l}}^{2} \Psi[\sigma](x, \theta)\right| \leq(2 \pi \ell)^{2} \mathcal{E}_{k l}^{\prime \prime}\|\sigma\|_{L^{1}\left(\mathbb{R}^{2}\right)}
$$

and

$$
\begin{aligned}
\left|\partial_{x_{k} x_{l}}^{2} \mu[\sigma](x)\right| \leq & \frac{1}{D} 2 \pi(2 \pi \ell)^{2} \mathcal{E}_{k l}^{\prime \prime}\|\sigma\|_{L^{1}\left(\mathbb{R}^{2}\right)} e^{c \mathcal{E}\|\sigma\|_{L^{1}\left(\mathbb{R}^{2}\right)}} \frac{1}{(2 \pi)^{2}} e^{2 c \mathcal{E}\|\sigma\|_{L^{1}\left(\mathbb{R}^{2}\right)}} \\
& +\frac{1}{D^{2}} 2 \pi(2 \pi \ell)^{2} \mathcal{E}_{k}^{\prime}\|\sigma\|_{L^{1}\left(\mathbb{R}^{2}\right)}(2 \pi \ell)^{2} \mathcal{E}_{l}^{\prime}\|\sigma\|_{L^{1}\left(\mathbb{R}^{2}\right)} e^{c \mathcal{E}\|\sigma\|_{L^{1}\left(\mathbb{R}^{2}\right)}} \frac{1}{(2 \pi)^{2}} e^{2 c \mathcal{E}\|\sigma\|_{L^{1}\left(\mathbb{R}^{2}\right)}} \\
& +\frac{2}{D} 2 \pi(2 \pi \ell)^{2} \mathcal{E}_{l}^{\prime}\|\sigma\|_{L^{1}\left(\mathbb{R}^{2}\right)} e^{c \mathcal{E}\|\sigma\|_{L^{1}\left(\mathbb{R}^{2}\right)}} \frac{1}{2 \pi} e^{c \mathcal{E}\|\sigma\|_{L^{1}\left(\mathbb{R}^{2}\right)}} \frac{c}{2 \pi} \mathcal{E}_{k}^{\prime}\|\sigma\|_{L^{1}\left(\mathbb{R}^{2}\right)} e^{3 c \mathcal{E}\|\sigma\|_{L^{1}\left(\mathbb{R}^{2}\right)}} \\
\leq & \left(\frac{c}{2 \pi} \mathcal{E}_{k l}^{\prime \prime}\|\sigma\|_{L^{1}\left(\mathbb{R}^{2}\right)}+\mathcal{E}_{k}^{\prime} \mathcal{E}_{l}^{\prime}\|\sigma\|_{L^{1}\left(\mathbb{R}^{2}\right)}^{2} \frac{3 c^{2}}{2 \pi}\right) e^{5 c \mathcal{E}\|\sigma\|_{L^{1}\left(\mathbb{R}^{2}\right)}}
\end{aligned}
$$

\section{Proof of Lemma 4.3}

Estimate (4.5) is a direct consequence of

$$
\iint_{\mathbb{R}^{2}}|(E * \sigma)(x)| \mathrm{d} x \leq\|E\|_{L^{1}\left(\mathbb{R}^{2}\right)}\|\sigma\|_{L^{1}\left(\mathbb{R}^{2}\right)} .
$$

We obtain (4.6) and (4.8) similarly. Next, we write

$$
\begin{aligned}
\iint_{\mathbb{R}^{2}}\left|\partial_{x_{k}} \mu[\sigma](x)\right| \mathrm{d} x & \leq \frac{2 \pi}{D} \iint_{\mathbb{R}^{2}}\left|\partial_{x_{k}} \Psi[\sigma](x, \theta)\right| \mathrm{d} x e^{c \mathcal{E}\|\sigma\|_{L^{1}\left(\mathbb{R}^{2}\right)}} \frac{e^{2 c \mathcal{E}}}{(2 \pi)^{2}}\|\sigma\|_{L^{1}\left(\mathbb{R}^{2}\right)} \\
& \leq \frac{c}{2 \pi} e^{3 c \mathcal{E}\|\sigma\|_{L^{1}\left(\mathbb{R}^{2}\right)}\left\|\partial_{x_{k}} E\right\|_{L^{1}\left(\mathbb{R}^{2}\right)}\|\sigma\|_{L^{1}\left(\mathbb{R}^{2}\right)}}
\end{aligned}
$$

It proves (4.7). Eventually, we have

$$
\begin{aligned}
& \iint_{\mathbb{R}^{2}}\left|\partial_{x_{k} x_{m}}^{2} \mu[\sigma](x)\right| \mathrm{d} x \\
\leq & \frac{1}{D} \int_{0}^{2 \pi} \iint_{\mathbb{R}^{2}}\left|\partial_{x_{k} x_{m}}^{2} \Psi[\sigma](x, \theta)\right| \mathrm{d} x \mathrm{~d} \theta e^{c \mathcal{E}\|\sigma\|_{L^{1}\left(\mathbb{R}^{2}\right)}} \frac{1}{(2 \pi)^{2}} e^{2 c \mathcal{E}\|\sigma\|_{L^{1}\left(\mathbb{R}^{2}\right)}} \\
& +\frac{1}{D^{2}} \int_{0}^{2 \pi} \iint_{\mathbb{R}^{2}}\left|\partial_{x_{k}} \Psi[\sigma](x, \theta)\right| \mathrm{d} x \mathrm{~d} \theta c D \mathcal{E}_{m}^{\prime}\|\sigma\|_{L^{1}\left(\mathbb{R}^{2}\right)} e^{c \mathcal{E}\|\sigma\|_{L^{1}\left(\mathbb{R}^{2}\right)}} \frac{1}{(2 \pi)^{2}} e^{2 c \mathcal{E}\|\sigma\|_{L^{1}\left(\mathbb{R}^{2}\right)}} \\
& +\frac{2}{D} \int_{0}^{2 \pi} \iint_{\mathbb{R}^{2}}\left|\partial_{x_{l}} \Psi[\sigma](x, \theta)\right| \mathrm{d} x \mathrm{~d} \theta e^{c \mathcal{E}\|\sigma\|_{L^{1}\left(\mathbb{R}^{2}\right)}} \frac{1}{2 \pi} e^{c \mathcal{E}\|\sigma\|_{L^{1}\left(\mathbb{R}^{2}\right)}} \frac{c}{2 \pi} \mathcal{E}_{k}^{\prime}\|\sigma\|_{L^{1}\left(\mathbb{R}^{2}\right)} e^{3 c \mathcal{E}\|\sigma\|_{L^{1}\left(\mathbb{R}^{2}\right)}}
\end{aligned}
$$




$$
\begin{aligned}
\leq & \frac{1}{D} c^{2} D^{2}\left\|\partial_{x_{k} x_{m}}^{2} E\right\|_{L^{1}\left(\mathbb{R}^{2}\right)}\|\sigma\|_{L^{1}\left(\mathbb{R}^{2}\right)} \frac{1}{2 \pi} e^{3 c \mathcal{E}\|\sigma\|_{L^{1}\left(\mathbb{R}^{2}\right)}} \\
& +\frac{1}{D^{2}} c D\left\|\partial_{x_{k}} E\right\|_{L^{1}\left(\mathbb{R}^{2}\right)}\|\sigma\|_{L^{1}\left(\mathbb{R}^{2}\right)} c D \mathcal{E}_{m}^{\prime}\|\sigma\|_{L^{1}\left(\mathbb{R}^{2}\right)} \frac{1}{2 \pi} e^{3 c \mathcal{E}\|\sigma\|_{L^{1}\left(\mathbb{R}^{2}\right)}} \\
& +\frac{2}{D} c D\left\|\partial_{x_{m}} E\right\|_{L^{1}\left(\mathbb{R}^{2}\right)}\|\sigma\|_{L^{1}\left(\mathbb{R}^{2}\right)} \frac{c}{2 \pi} \mathcal{E}_{k}^{\prime}\|\sigma\|_{L^{1}\left(\mathbb{R}^{2}\right)} e^{5 c \mathcal{E}\|\sigma\|_{L^{1}\left(\mathbb{R}^{2}\right)}}
\end{aligned}
$$

\section{References}

[1] P. Amorim. Modeling ant foraging: a chemotaxis approach with pheromones and trail formation. J. Theor. Biol., 385:160-173, 2015.

[2] P. Amorim, T. Goudon, and F. Peruani. An ant navigation model based on Weber's law. J. Math. Biol., 78(4):943-984, 2019.

[3] L. Barberis and F. Peruani. Large-scale patterns in a minimal cognitive flocking model: incidental leaders, nematic patterns, and aggregates. Phys. Rev. Lett., 117:248001, 2016.

[4] J. A. Carrillo, M. Fornasier, G. Toscani, and F. Vecil. Particle, kinetic, and hydrodynamic models of swarming, pages 297-336. Modeling and Simulation in Science, Engineering and Technology. Birkhauser, 2010.

[5] G.-Q. Chen and M. Rascle. Initial layers and uniqueness of weak entropy solutions to hyperbolic conservation laws. Arch. Rat. Mech. Anal., $153(3): 205-220,2000$.

[6] R. Colombo, M. Garavello, and M. Lécureux-Mercier. A class of nonlocal models for pedestrian traffic. Math. Mod. and Meth. in Appl. Sci., 22(4):1150023, 2012.

[7] R. Colombo, M. Mercier, and M. Rosini. Stability and total variation estimates on general scalar balance laws. Commun. Math. Sci., 7(1):37-65, 2009.

[8] F. Cucker and S. Smale. Emergent behavior in flocks. IEEE Trans. Automat. Control, 52:852-862, 2007.

[9] F. Cucker and S. Smale. On the mathematics of emergence. Japan. J. Math., 2:197-227, 2007.

[10] P. Degond. Mathematical models of collective dynamics and selforganization. In Proceedings of the International Congress of Mathematicians, Rio de Janeiro, Brazil, 2018.

[11] P. Degond, A. Frouvelle, and J.-G. Liu. Macroscopic limits and phase transition in a system of self-propelled particles. J. Nonlinear Sc., 23(3):427$456,2013$. 
[12] P. Degond and S. Motsch. Continuum limit of self-driven particles with orientation interaction. Math. Models Methods Appl. Sci., 18(1):1193-1215, 2008 .

[13] M. R. D'Orsogna, Y. L. Chuang, A. L. Bertozzi, and L. S. Chayes. Selfpropelled particles with soft-core interactions: Patterns, stability, and collapse. Phys. Rev. Lett., 96(10):104302, 2006.

[14] R. Erban and H. G. Othmer. From individual to collective behavior in bacterial chemotaxis. SIAM J. Appl. Math., 65:361-391, 2004.

[15] M. A. Fontelos, A. Garnier, and M. Vela-Perez. From individual to collective dynamics in Argentine ants (linepithema humile). Math. Biosc., 262:56-64, 2015.

[16] R. Grossmann, L. Schimansky-Geier, and P. Romanczuk. Self-propelled particles with selective attraction-repulsion interaction: from microscopic dynamics to coarse-grained theories. New J. Phys., 15:085014, 2013.

[17] S.-Y. Ha and E. Tadmor. From particle to kinetic and hydrodynamic descriptions of flocking. Kinet. Relat. Models, 1(3):415-435, 2008.

[18] S. N. Kruzhkov. First order quasilinear equations in several independent variables. Math. Sb., 123:228-255, 1970. English translation in Math. USSR Sbornik 10 (1970), pp. 217-243.

[19] M. Lécureux-Mercier. Improved stability estimates on general scalar balance laws. J. Hyper. Diff. Eq., 8(4):727-757, 2011.

[20] L. Gómez Nava, R. Grossmann, and F. Peruani. Markov robots: minimal navigation strategies for active particles. Phys. Rev., 97:042604, 2018.

[21] J. Nieto, F. Poupaud, and J. Soler. About uniqueness of weak solutions to first order quasi-linear equations. Math. Models Methods Appl. Sci., 12(11):1599-1605, 2002.

[22] O. A. Oleŭnik. Discontinuous solutions of non-linear differential equations. Uspehi Mat. Nauk (N.S.), 12(3(75)):3-73, 1957. English translation in Amer. Math. Soc. Transl., Ser. 2, 26 (1963) pp. 95-192.

[23] J. K. Parrish, S. V. Viscido, and D. Grünbaum. Self-organized fish schools: an examination of emergent properties. Biological Bulletin, 202:296-305, 2002.

[24] S. D. Ryan. A model for collective dynamics in ant raids. J. Math. Biol., 72:1579-1606, 2015.

[25] Camazine S., Deneubourg J.-L., Franks N. R., J. Sneyd, G. Theraulaz, and E. Bonabeau. Self-organization in biological systems. Princeton studies in complexity. Princeton University Press, 2001. 
[26] J. Toner and Y. Tu. Flocks, herds, and schools: A quantitative theory of flocking. Phys. Rev. E, 58:4828-4858, 1998.

[27] A. Vasseur. Strong traces for solutions to multidimensional scalar conservation laws. Arch. Rat. Mech. Anal., 160(3):181-193, 2001.

[28] T. Vicsek, A. Czirok, E. Ben-Jacob, I. Cohen, and O. Shochet. Novel type of phase transition in a system of self-driven particles. Phys. Rev. Lett., 75:1226-1229, 1995.

[29] T. Vicsek and A. Zafeiris. Collective motion. Phys. Reports, 517(3-4):71$140,2012$. 